\title{
Post-Encoding Amygdala-Visuosensory Coupling Is Associated with Negative Memory Bias in Healthy Young Adults
}

\author{
Sarah M. Kark and Elizabeth A. Kensinger \\ Department of Psychology, Boston College, Chestnut Hill, Massachusetts 02467
}

The amygdala is well documented as the critical nexus of emotionally enhanced memory, yet its role in the creation of negative memory biases, better memory for negative compared with positive stimuli, has not been clarified. Although prior work suggests valence-specific effects at the moment of "online" encoding and retrieval, with enhanced visuosensory processes supporting negative memories in particular, here we tested the novel hypothesis that the amygdala engages with distant cortical regions after encoding in a manner that predicts inter-individual differences in negative memory biases in humans. Twenty-nine young adults (males and females) were scanned while they incidentally encoded negative, neutral, and positive scenes, each preceded by a line-drawing sketch of the scene. Twenty-four hours later, participants were scanned during an Old/New recognition memory task with only the line-drawings presented as retrieval cues. We replicated and extended our prior work, showing that enhanced online visuosensory recapitulation supports negative memory. Critically, resting-state scans flanked the encoding task, allowing us to show for the first time that individual differences in "off-line" increases in amygdala resting-state functional connectivity (RSFC) immediately following encoding relate to negative and positive memory bias at test. Specifically, post-encoding increases in amygdala RSFC with visuosensory and frontal regions were associated with the degree of negative and positive memory bias, respectively. These findings provide new evidence that valence-specific negative memory biases can be linked to the way that sensory processes are integrated into amygdala-centered emotional memory networks.

Key words: amygdala; emotion; memory; post-encoding; resting state; valence

Significance Statement

Decades of research has placed the amygdala at the center of the emotional memory network. Despite the clinical importance of disproportionate memory for negative compared with positive events, it is not known whether post-encoding increases in amygdala-cortical coupling, possibly reflective of early consolidation processes, bear any influence on the degree or direction of such emotional memory biases. We demonstrate that, across participants, increases in post-encoding amygdala coupling with visuosensory and frontal regions are associated with more pronounced negative and positive memory biases, respectively. These findings provide the first evidence linking post-encoding amygdala modulation to the degree of negative or positive memory bias, emphasizing the need for valence-based accounts of the amygdala's role in emotional memory.

\section{Introduction}

We tend to remember the good and bad events in our lives long past the time when trivial events have slipped from our memo-

\footnotetext{
Received Nov. 4, 2018; revised Jan. 27, 2019; accepted Feb. 5, 2019.

Author contributions: S.M.K. and E.A.K. designed research; S.M.K. performed research; S.M.K. and E.A.K. analyzed data; S.M.K. and E.A.K. wrote the paper.

This work was supported by NSF Grant BCS 1539361 E.A.K. and Jessica D. Payne, NSF-GRFP DGE1258923 to S.M.K., pre-doctoral NRSA fellowship 5F31MH113304-02 to S.M.K., Sigmi Xi Grant-in-Aid of Research to S.M.K., and NIH shared instrumentation Grant S100D020039 (Harvard Center for Brain Science). SMS-BOLD sequences were received by the University of Minnesota Center for Magnetic Resonance Research. We thank Tala Berro, Ryan Daley, Kevin Frederiks, Sandry Garcia, Olivia Hampton, Lauren Lu, and Stephanie Sherman for assistance with participant recruitment and data collection and processing; Ehri Rhyu for helpful discussions of the mediation analysis; and Jaclyn Ford for her thoughtful comments and helpful discussions of this work. Further details of the recapitulation
}

ries, but recent work suggests negative and positive memories are not always created equally in brain or behavior (Bowen et al., 2018). Despite the clinical relevance of understanding how disproportionate memory for negative events over positive events, a cognitive risk factor for depression (Gerritsen et al., 2012), arises from individual differences in neural memory processes, these relationships have yet to be tested empirically. The bulk of task-

replication results and exploratory moderated mediation analysis can be obtained by contacting the corresponding author directly. This work was included in the dissertation of S.M.K.

The authors declare no competing financial interests.

Correspondence should be addressed to Sarah M. Kark at kark@bc.edu.

https://doi.org/10.1523/JNEUROSCI.2834-18.2019

Copyright $\odot 2019$ the authors 
based fMRI work on emotional memory has focused on the encoding of negative stimuli, with a focus on the amygdala, hippocampus, ventral visual stream, and prefrontal cortex (Murty et al., 2011). However, studies comparing memory for positive and negative events have suggested that although the amygdala is engaged by both valences (Hamann et al., 1999), the effect of arousal on the targets of amygdala-cortical coupling during encoding can depend on valence (Mickley Steinmetz et al., 2010). Our prior research has shown valence-specific memory effects during retrieval, with greater retrieval-related reactivation of encoding processes in visuosensory regions for negative events relative to positive and neutral ones (Kark and Kensinger, 2015; Bowen and Kensinger, 2017a,b), evidence that contributed to our proposed valence-based model of emotional memory (Bowen et al., 2018).

Decades of animal and human work in support of the modulation hypothesis of amygdala function has shown that the amygdala is the critical "nexus" of emotional memory formation and consolidation because of its ability modulate neural processes in medial temporal lobe (MTL) regions and distant cortical regions (Cahill and McGaugh, 1998; McGaugh, 2000; Hermans et al., 2014), including visual cortices (Dringenberg et al., 2004; Vuilleumier et al., 2004). Feedback projections from the amygdala to almost all levels of visual cortex are thought to enhance their response during emotional situations (Amaral et al., 2003; Silverstein and Ingvar, 2015) and likely continue to influence memory processes after the initial encoding experience itself (Müller and Pilzecker, 1900; McGaugh, 2005). Thus, the amygdala is well positioned to exert long-lasting negative memory enhancing effects in visuosensory regions.

Most of the work described in the previous paragraphs has monitored "online" memory processes to reveal neural mechanisms that support emotional memory formation and retrieval during task. However, "off-line" post-encoding resting-state functional connectivity (RSFC) analysis has become increasingly used to reveal links between early consolidation processes and memory performance (Tambini et al., 2010; Hermans et al., 2017; Murty et al., 2017). Here, we use this approach in the emotional episodic memory realm to investigate the links between postencoding changes in amygdala-cortical RSFC and behavioral measures of emotional memory bias across participants, an approach that has the potential to unveil off-line early consolidation processes that differentially predict long-term negative and positive memory outcomes.

We adjudicated between arousal-based and valence-based accounts of emotional modulation of early consolidation processes. An arousal-based account of emotional memory would predict the same link between post-encoding increases in amygdala RSFC and enhanced memory for negative and positive stimuli, whereas our valence-based account would predict a strong link between post-encoding increases in amygdala-visuosensory RSFC and negative memory biases specifically, with a link to neutral memory falling between negative and positive. Based on previous work (Mickley Steinmetz et al., 2010), a secondary hypothesis was that amygdala-frontal RSFC enhancements would relate to positive memory biases.

Here, resting-state fMRI scans flanked the encoding scan and preceded the recognition scan of an emotional recognition memory paradigm with a $24 \mathrm{~h}$ study-test delay. Participants incidentally encoded line-drawings of scenes (negative, positive, and neutral), each followed by the full image. At test, only old and new line-drawings were presented for an Old/New judgment. We root our novel test of the links between post-encoding increases in amygdala RSFC and valence-specific memory biases across participants in a replication-extension of our prior work (Kark and Kensinger, 2015) showing enhanced visuosensory recapitulation for negative memories.

\section{Materials and Methods Participants}

Thirty-three participants were recruited to participate in the control (no induced stress) condition of a larger study examining the effects of stress and sleep on emotional memory. All participants were right-handed, native English speakers between the ages of 18 and 29, with normal or corrected-to-normal vision and with no reported history of head injury, learning disorders, neurologic or psychiatric problems, or current medications affecting the central nervous system. Participants were screened for MRI environment contradictions before entering the scanner. The Boston College Institutional Review Board approved this study and written informed consent of study procedures was obtained from all participants. Participants were compensated $\$ 25 / \mathrm{h}$ for their participation.

For inclusion in this set of analyses, participants needed to have usable data from encoding and retrieval fMRI scans, including above-chance recognition memory performance, as well as at least $5 \mathrm{~min}$ of usable RSFC data from pre-encoding and post-encoding (Waheed et al., 2016). (Although additional measures were gathered as part of the larger study, they were not examined for this analysis and therefore were not required for data inclusion.) Four participants were excluded from all of the present analyses: one because of chance-level memory performance (an overall $d^{\prime}$ value $<0$; male, 25), one because of a brain structure anomaly (female, 23), one did not undergo a post-encoding RSFC scan because of time constraints and additionally did not have enough trials per condition for task fMRI analyses (female, 21), and one participant (male, 20) had excessive motion across resting-state fMRI scans, resulting in $<5$ min of useable data for each resting scan. The final sample for the RSFC analyses was 29 participants ages $18-29\left(14\right.$ female, $\mathrm{M}_{\text {age }}=22.3, \mathrm{SD}_{\text {age }}=$ 2.8 ). For the task-based fMRI analyses of subsequent memory and retrieval success, seven additional participants ( 3 females) were excluded because they did not have an ample number of trials across all of the memory conditions by valence (trials count requirement $\geq 8$ ). The final sample for the memory task-fMRI analyses was 22 participants ( $11 \mathrm{fe}-$ males, $\mathrm{M}_{\text {age }}=22.2, \mathrm{SD}_{\text {age }}=2.8$ ). However, to examine how brainbehavior patterns from the 22 participant sample expand to the larger sample (with possibly noisier activation estimations), the data for these 7 participants will appear as open circles in the follow-up scatter plot for the memory retrieval fMRI analyses (see Fig. 2B).

\section{Experimental design}

Participants underwent fMRI scanning at the Harvard Center for Brain Science during both an incidental encoding task and a surprise recognition memory task $\sim 24 \mathrm{~h}$ later (Fig. $1 A$ shows a depiction of the timeline for acquisition of data).

\section{Encoding task}

The encoding task is depicted in Figure 1B. Study stimuli were 300 images selected from the Internal Affective Picture System (IAPS; Lang et al., 2008) database and nearly identical to the set used in Kark and Kensinger (2015). In brief, participants viewed 150 line-drawings of IAPS images ( 50 negative, 50 neutral, 50 positive, each for $1.5 \mathrm{~s}$ ), followed by the full-color photo of that line-drawing $(3 \mathrm{~s})$. As an incidental encoding task, participants made a button press to indicate whether they would "Approach" or "Back away" from each of the images. The negative and positive images were preselected using the normative data provided by IAPS (Lang et al., 2008) to ensure that the negative images were equally arousing $\left(M_{\text {neg }}=5.54, M_{\text {pos }}=5.43, t_{(198)}=1.32, p=0.19\right.$, independent samples $t$ test $)$ and of similar absolute valence $\left(M_{\text {neg }}=2.05, M_{\text {pos }}=2.07\right.$, $t_{(198)}=0.25, p=0.80$, independent samples $t$ test) as the positive images. The negative images were more arousing $\left(M_{\text {neut }}=3.25, t_{(198)}=23.95\right.$, $p<0.001$, independent samples $t$ test) and higher in absolute valence $\left(M_{\text {neut }}=0.42, t_{(198)}=19.27, p<0.001\right.$, independent samples $t$ test $)$ than the neutral images. Similarly, positive images were more arousing 
$t_{(198)}=22.97, p<0.001$, independent samples $t$ test $)$ and higher in absolute valence $\left(t_{(198)}\right.$ $=25.00, p<0.001$, independent samples $t$ test) than neutral images. Line-drawing versions of these IAPS images were created using in-house MATLAB scripts (Fig. $1 B, C$ shows examples of IAPS images and their line-drawings). Restingstate scans were collected immediately before and after the encoding task runs. During each of the three resting-state scans, the stimuli presentation computer monitor was turned off (i.e., no fixation cross was presented) and participants were instructed to relax with their eyes open and think about anything that came to mind. The eye-tracking camera was on throughout the resting-state scans and monitored by the experimenters to ensure that participants kept their eyes open for the majority of each rest scan.

\section{Recognition task}

Twenty-four hours after encoding, participants returned to the scanner for a surprise recognition memory task. After a pre-retrieval resting-state scan, participants were presented with all 150 of the old line-drawings ( $3 \mathrm{~s}$ each, jittered fixation 1.5-9 s) that they had seen during encoding randomly mixed with an equal number of new line-drawings (study lists were varied across participants such that studied line-drawings for some participants were the new line-drawings for other participants). For each line-drawing, participants were instructed to use a $0-4$ scale to indicate in one decision whether the line-drawing was new (0) or, if old, how vividly they remembered the colorful photo $(1=$ "Not Vivid" to $4=$ "Extremely Vivid"). Here, we collapse across vividness ratings to compare old (1-4) to new (0) responses. To ensure participants understood that half of the line-drawings were old and half were new, we instructed them during the practice and instruction period that they should be pressing the 0 key "about half of the time". The use of line-drawing cues, as opposed to representation of the full-color IAPS images, allows us to (1) cue individual memories with less-emotionally laden visual cues, and (2) trigger memories while minimizing visual and emotion induction confounds at the time of retrieval.

\section{Post-recognition arousal and}

valence ratings

After the recognition scan, and outside of the scanner, participants completed post-scan ratings of arousal and valence (1-7 scales) of the 300 IAPS images. Results of these post-scan IAPS ratings confirmed that negative images were more arousing $\left(\mathrm{M}_{\text {neg }}=5.72, \mathrm{SD}_{\text {neg }}=0.61, \mathrm{M}_{\text {neut }}\right.$ $=4.0, \mathrm{SD}_{\text {neut }}=0.37, t_{(28)}=15.65, p<0.001$, paired samples $t$ test $)$ and higher in absolute valence $\left(\mathrm{M}_{\text {neg }}=2.20, \mathrm{SD}_{\text {neg }}=0.38, \mathrm{M}_{\text {neut }}=0.62\right.$, $\mathrm{SD}_{\text {neut }}=0.36, t_{(28)}=19.43, p<0.001$, paired samples $t$ test) than the neutral images. Positive images also were more arousing $\left(\mathrm{M}_{\mathrm{pos}}=4.60\right.$, $\mathrm{SD}_{\text {pos }}=0.84, t_{(28)}=4.40, p<0.001$, paired samples $t$ test $)$ and higher in absolute valence $\left(\mathrm{M}_{\text {pos }}=1.77, \mathrm{SD}_{\text {pos }}=0.37, t_{(28)}=19.23, p<0.001\right.$, paired samples $t$ test) than the neutral images. However, despite being equated for arousal based on IAPS normative data (see Encoding task) negative images were rated as more arousing $\left(t_{(28)}=6.09, p<0.001\right.$, paired samples $t$ test) and higher in absolute valence $\left(t_{(28)}=6.06, p<\right.$ 0.001 , paired samples $t$ test) than the positive images. The same pattern of

\section{A Scans used for current data analyses}

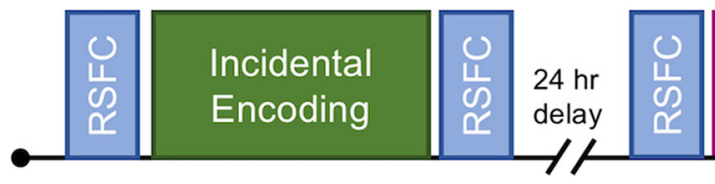

Surprise

Recognition

\section{B Incidental Encoding Task}

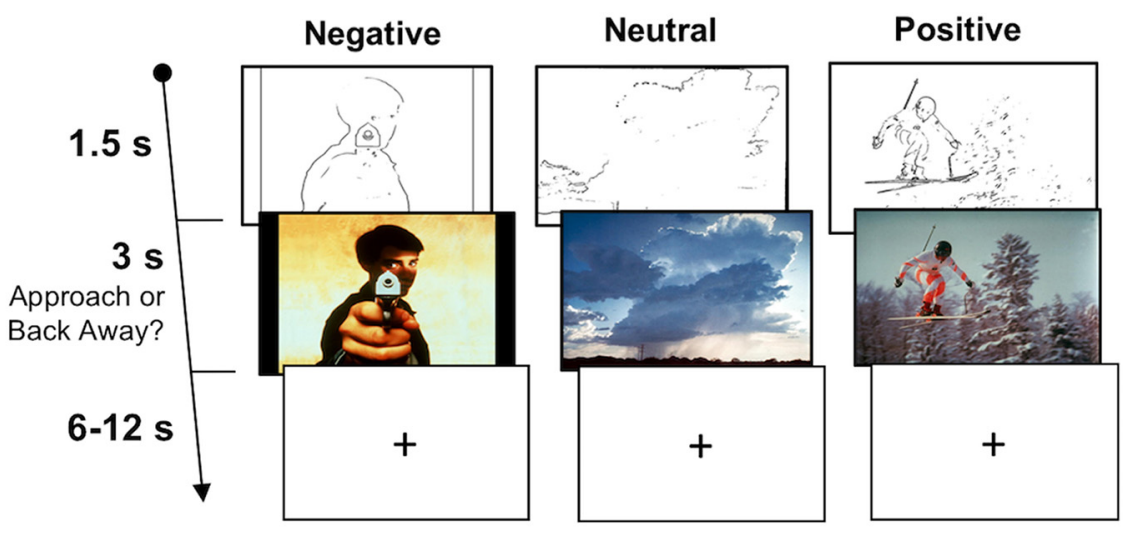

\section{Sample Recognition Stimuli}

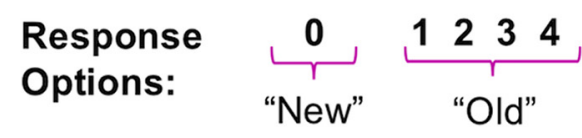

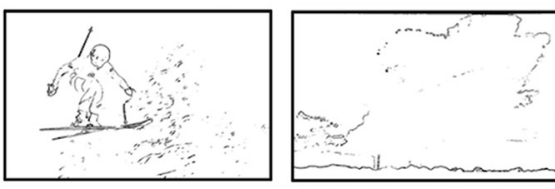

Previously

Studied

(Old)
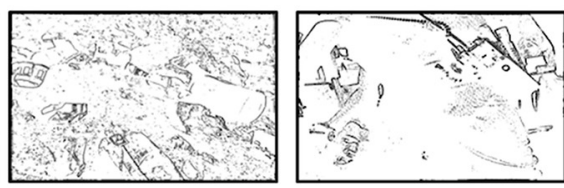

Not previously

Studied

(New)

Figure 1. A, Participants were scanned over the course of $2 \mathrm{~d}$ during an incidental encoding and a $24 \mathrm{~h}$ surprise recognition memory task. RSFC scans were acquired before and after incidental encoding, as well as directly before retrieval. $\boldsymbol{B}$, Sample ing and an equal number of new line-drawings were presented one at time. For each item, participants had $3 \mathrm{~s}$ to rate whether a line-drawing was "Old" (1-4) or "New" (0), followed by a jittered fixation period (1.5-9s). Sample recognition stimuli are shown, with the depicted study history listed below in the gray boxes.

results was observed in the subset of 22 participants in the memory task-based fMRI analyses.

Although the post-scan ratings of absolute valence and arousal were greater for negative stimuli, compared with positive stimuli, it is important to keep in mind that these ratings were made after participants had studied and retrieved the images, which could impact their valence and arousal strengths (e.g., perhaps these negative stimuli maintain their arousal even after multiple viewings, while positive stimuli show mitigation or habituation of arousal over repeated presentations). Nevertheless, in the fMRI data, valence-based patterns will be considered to occur when region-of-interest (ROI) analyses reveal a pattern of Negative greater than Neutral greater than Positive, not just Negative greater than Positive greater than Neutral, to ensure that they cannot simply be driven by lower arousal or absolute-valence in the positive images (because the positive images were rated as more arousing and of greater absolutevalence than the neutral images, as reported in the previous section). 
Moreover, for the replication fMRI analysis by Kark and Kensinger (2015), we show the recapitulation results from an analysis that included individual ratings of subjective arousal as a nuisance regressor in the fixed-effects models of encoding and retrieval. To ensure the reported across-subjects' effects were not driven by any valence differences of subjective arousal across participants (i.e., those with the greatest difference between negative and positive arousal ratings), we controlled for valence differences of arousal between-subjects (i.e., Arousal ${ }_{\text {neg-pos: }}$ average negative arousal ratings - average positive arousal ratings), where applicable.

\section{MRI acquisition}

Structural and functional images were acquired using a Siemens MAGNETOM Prisma 3T scanner with a 32-channel head coil. A localizer and auto-align scout were followed by collection of whole-brain $\mathrm{T}_{1}$-weighted anatomical images (MEMPRAGE, 176 slices, $1.0 \mathrm{~mm}^{3}$ voxels, TR $=2530$ $\mathrm{ms}$, flip angle $=7$ degrees, field-of-view $=256 \mathrm{~mm}$, base resolution $=$ 256). The functional images were acquired using simultaneous multislice blood oxygen level-dependent (SMS-BOLD) scan sequences (Barth et al., 2016) provided by the Center for Magnetic Resonance Research at University of Minnesota (Feinberg et al., 2010; Moeller et al., 2010; Xu et al., 2013). All $\mathrm{T}_{2}$-weighted EPI images were acquired in an interleaved fashion and included the whole brain, with the slices aligned $25^{\circ}$ above the anterior commissure-posterior commissure line in the coronal direction. The pulse sequences for the encoding and retrieval task-fMRI scans (69 slices, $\mathrm{TR}=1500 \mathrm{~ms}, 2.0 \mathrm{~mm}^{3}$ isotropic voxels, $\mathrm{TE}=28 \mathrm{~ms}$, flip angle $=75$ degrees, field-of-view $=208 \mathrm{~mm}$, base resolution $=104$, multiband acceleration factor $=3$ ) differed from for the resting-state EPI sequence $\left(\mathrm{TR}=650 \mathrm{~ms}\right.$, 64 slices, $2.3 \mathrm{~mm}^{3}$ voxels, $\mathrm{TE}=34.8 \mathrm{~ms}$, flip angle $=52$ degrees, field-of-view read $=207 \mathrm{~mm}$, multiband acceleration factor $=8$, base resolution $=90$ ).

\section{Statistical analysis}

\section{Memory performance and emotional memory bias}

Effects of valence on memory performance [as calculated by $d^{\prime}=z$ (hit rate) $-z$ (false alarm rate)] was tested using repeated-measures ANOVA with a factor of valence. Negative memory was bias (Negative $d^{\prime}-$ Positive $d^{\prime}$ ) and Positive memory bias (Positive $d^{\prime}-$ Negative $d^{\prime}$ ) were calculated for each participant. Given that memory biases were calculated as difference scores, the fMRI data contrasts of interest were additionally masked where appropriate to ensure results were also correlated with memory performance of the single valence (e.g., Negative $d^{\prime}$ alone), and thus not driven by an inverse relationship with the other valence (e.g., Positive $d^{\prime}$ ). Throughout the remainder of the analyses, memory performance refers to the $d^{\prime}$ score for a given valence category, whereas memory bias is the difference between $d^{\prime}$ scores between negative and positive stimuli.

\section{fMRI preprocessing}

Memory task-based fMRI. fMRI images from the encoding and retrieval scans were preprocessed and analyzed using SPM8 (Wellcome Department of Cognitive Neurology, London, UK) implemented in MATLAB 2014a. All functional images were reoriented, realigned, coregistered, and spatially normalized to the Montreal Neurological Institute (MNI) template (resampled at $3 \mathrm{~mm}$ during segmentation and written at $2 \mathrm{~mm}$ during normalization), and smoothed using a $6 \mathrm{~mm}$ isotropic Gaussian kernel. The first four scans of each run were discarded to account for scanner equilibrium effects. Global mean intensity, rotation, and translation motion outliers were identified using Artifact Detection Tools (ART; available at http://www.nitrc.org/projects/artifact_detect). Global mean intensity outliers were defined as scans with a global mean intensity that differed by $>3$ SD from the mean. Acceptable motion parameters were set to $3 \mathrm{~mm}$ for translation and $3^{\circ}$ for rotation. Framewise displacement (FWD), the average rotation and translation parameter differences from scan to scan, using weight scaling (Power et al., 2012), was calculated for each participant. Individual scan runs were eliminated if $>5 \%$ of the time points were identified as having (1) an FWD value $>0.5 \mathrm{~mm}$ (Power et al., 2012), and (2) $>3 \mathrm{~mm}$ of movement $/ 3^{\circ}$ rotation. In total, four scan runs were excluded from the encoding analyses ( 2 encoding runs for 1 participant, and 1 encoding run each for 2 other participants) and five scan runs were excluded from retrieval analyses (1 run from 5 different participants). One participant was completely removed from the memory task-based fMRI analysis because 3 of their 6 retrieval runs showed excessive head motion based on these thresholds, resulting in too few trials for analysis. Participants were required to still have an ample number $(\geq 8)$ of each response type (hits and misses by valence) after any individual scan runs were removed because of motion.

Resting-state fMRI. Resting-state scans were preprocessed and denoised using the CONN Toolbox (Whitfield-Gabrieli and NietoCastanon, 2012; http://www.nitrc.org/projects/conn; RRID:SCR_009550) implemented in MATLAB 2015a and SPM12. To ensure the scanner had reached a steady state, the first six time points of each RSFC scan were discarded (Waheed et al., 2016). Functional scans were then realigned and unwarped, centered, segmented, normalized to MNI space, and smoothed with an $8 \mathrm{~mm}$ Gaussian smoothing kernel. Functional data were resampled to $2 \mathrm{~mm}$ isotropic voxels. Conservative functional outlier detection settings were used during the ART-based identification of outlier scans for scrubbing (global signal $z$ value threshold of 3 , subjectmotion threshold of $0.3 \mathrm{~mm}$ ). Preprocessed resting scans for each participant were linearly detrended and a commonly used bandpass filter $(0.008-0.09 \mathrm{~Hz})$ was applied after regression to isolate low-frequency fluctuations characteristic of resting-state fMRI and attenuate signals outside of that range (Fox et al., 2005, 2006; Waheed et al., 2016). White matter and CSF noise sources were removed using the CONN Toolbox aCompCor method for noise removal. After artifact scrubbing, all participants included in the present analyses had at least $5.2 \mathrm{~min}$ $(\mathrm{M}=7.6 \mathrm{~min})$ of useable time points for each of the RSFC scans. One additional participant (male, 28), also excluded from the memory task-fMRI analyses, was excluded from the follow-up analysis of preretrieval RSFC because of excessive motion resulting in $<5$ min of pre-retrieval RSFC data.

\section{Memory task- $\mathrm{fMRI}$ analyses}

General linear models. For each participant, first-level models were created for encoding and retrieval separately. For both encoding and retrieval, each model contained 12 regressors-of-interest: hits and misses by the three valence categories, each with a parametric modulator for item reaction times to control for the time to make the Approach/Back Away decision (during encoding) or the memory judgment (during retrieval). Retrieval models additionally included correct rejections and false alarms, collapsed across valence. To control for low-level visual confounds, an additional nuisance regressor column contained itemlevel visual statistic information for the TRs that the images were on the screen (i.e., average image saliency for each IAPS image for the encoding models and edge density of the line-drawings for the retrieval models). Image saliency for each IAPS photo was calculated using the Saliency Toolbox (Itti and Koch, 2001) and the edge density each line-drawing image was calculated as the proportion of black pixels within the image frame using MATLAB. Finally, seven motion regressors (FWD; $x, y, z$; roll, pitch, yaw) were added before the linear drift regressors.

Analyses to test for replication of encoding-to-retrieval overlap. For encoding and retrieval separately, full-factorial $2 \times 3$ ANOVAs at the random-effects level were created with factors of memory (hits, misses) and valence (negative, neutral, positive). Encoding-to-retrieval overlap (or "recapitulation") effects were operationalized like they were in the original study (Kark and Kensinger, 2015) as the spatial overlap of regions that exhibit differences because of memory at encoding [Wagner et al., 1999; Paller and Wagner, 2002; Hits > Misses, differences due to memory $(\mathrm{Dm})$ effects] and Retrieval Success Activity at retrieval (Hits $>$ Misses). Practically, the encoding-to-retrieval overlap (or recapitulation) maps are a conjunction of encoding and retrieval maps (Encoding Dm $\cap$ Retrieval Success) executed separately for each valence (e.g., Encoding Negative Dm $\cap$ Negative Retrieval Success). Encoding-to-retrieval overlap maps were created for negative, neutral, and positive recapitulation effects separately (Fig. $2 A$, activity in red, white, and blue, respectively). The same approach was taken to analyze random-effects level recapitulation effects while controlling for subjective ratings of arousal in the 
fixed-effects models (Fig. 2A, activity in magenta and cyan for negative and positive stimuli controlling for arousal, respectively).

Relations between valence-specific retrieval success activity and individual differences in emotional memory bias. Here, we conduct the first individual differences examination for links between valence-specific activity at the moment of successful retrieval and corresponding valence-specific memory biases. To test whether those participants who recognized more negative stimuli than positive stimuli were those with greater retrieval success activity in visuosensory regions, a whole-brain onesample $t$ test was used to demarcate regions that showed a correlation between the firstlevel parameter estimates of Negative-Biased Retrieval Success Activity (e.g., Negative Retrieval Success $>$ Positive Retrieval Success) and the magnitude the negative memory bias (e.g., Negative $d^{\prime}-$ Positive $d^{\prime}$ ). To ensure the resulting clusters were not driven by the inverse relationship for positive memory effects, the resulting map was also inclusively masked with the results of an additional one-sample $t$ test to demarcate regions that also showed a correlation between Negative Retrieval Success Activity and Negative $d^{\prime}$ (held at a reduced threshold of $p<0.05$ ). We conducted a similar analysis to assess links between Positive-Biased Retrieval Success Activity and positive memory bias.

\section{Resting-state fMRI analyses}

Whole-brain seed-to-voxel RSFC were conducted using left and right amygdala seeds from a maximum probability atlas of the human brain (Hammers et al., 2003). These analyses produced Fisher $r$-to- $Z$ transformed whole-brain maps of pre-encoding, postencoding, and pre-retrieval amygdala RSFC for each participant. First-level $r$-to- $Z$ RSFC maps outputted from CONN were entered into a factorial in SPM8 for group analysis with one factor (i.e., condition) with two levels (preencoding, post-encoding). To test for valencespecific memory biases, we entered Negative $d^{\prime}$, Neutral $d^{\prime}$, and Positive $d^{\prime}$ into the model as covariates set to interact with the condition factor.

Baseline amygdala RSFC. We began the RSFC analyses with a replication of baseline amygdala RSFC patterns. We first examined group-level pre-encoding $\left(Z_{\text {Pre }}\right)$ amygdala RSFC maps as a comparison to prior work characterizing RSFC networks of the amygdala. For comparison with previous work reporting few changes in the amygdala RSFC following emotion picture viewing (Geissmann et al., 2018), next we examined overall pre- to postencoding increases in amygdala $\operatorname{RSFC}\left(Z_{\text {Post }}>\right.$ $Z_{\text {Pre }}$ masked with $Z_{\text {Post }}$ thresholded at $p<$ $0.005)$. These first two analyses were used to establish our group findings before exploring the novel inter-individual difference questions central to the purpose of the current study.

Post-encoding amygdala RSFC and emotional memory biases. The central purpose of the RSFC analysis was to test the hypothesis that

\section{A Replication of Encoding-to-Retrieval Overlap} Encoding $\cap$ Retrieval: Hits > Misses

$\begin{array}{ll}\text { Negative } & \square \text { Neutral } \\ \text { Arousal controlled } & \text { Positive } \\ & \square \text { Arousal controlled }\end{array}$

$\star$ Overlap with Kark and Kensinger (2015)

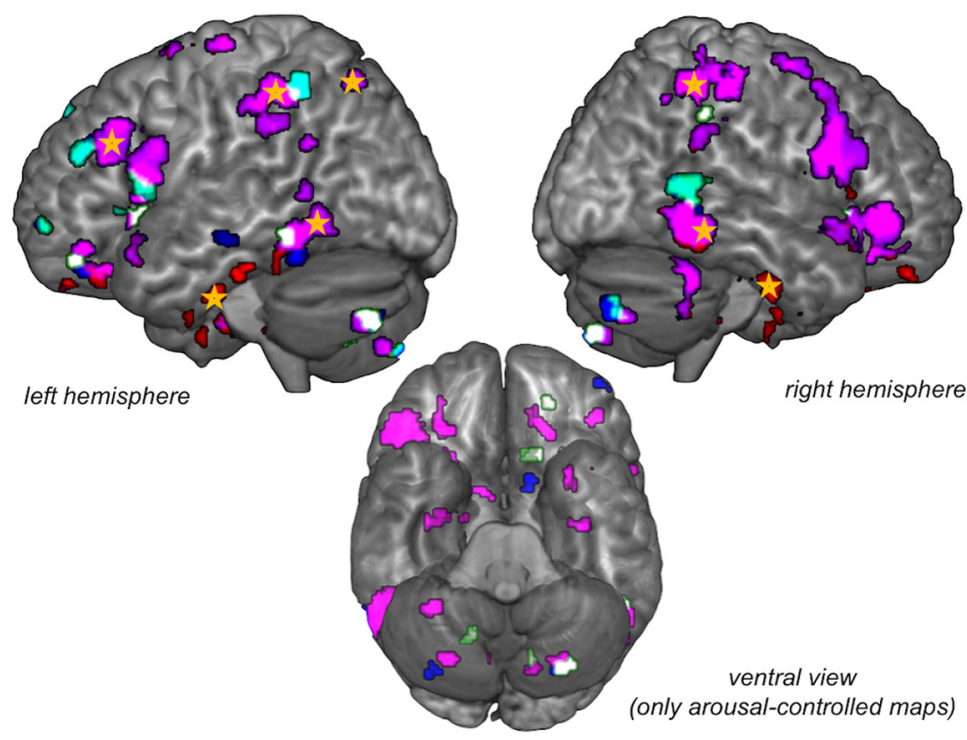

\section{B Retrieval activity and negative memory bias}

\section{Negative > Positive Retrieval Success Activity: $\square$ Correlated with Negative memory bias \\ $\square$ Masked with Negative Retrieval Success correlated with Negative d'}
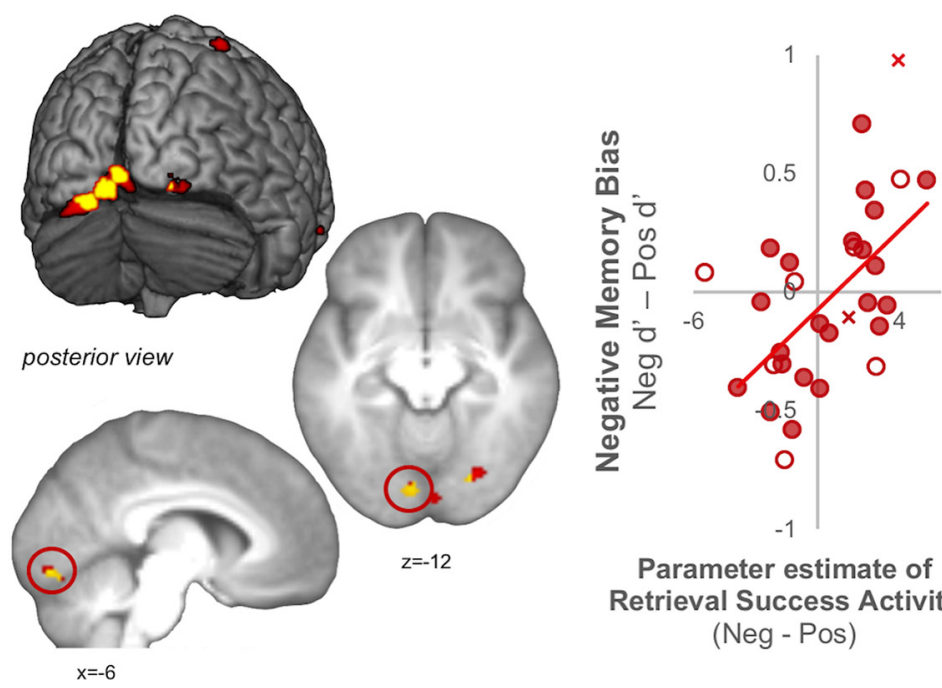

Parameter estimate of Retrieval Success Activity (Neg - Pos)

Figure 2. A, Replication of group-level whole-brain encoding-to-retrieval overlap effects for negative stimuli are plotted in red, with regions that directly overlap with clusters from our prior study (Kark and Kensinger, 2015) demarcated using yellow star symbols. Regions that survived controlling for item-level subjective arousal from the post-scan ratings are shown in magenta for negative memories and cyan for positive memories. $\boldsymbol{B}$, Whole-brain correlations between individual differences in Negative $>$ Positive Retrieval Success Activity and negative memory bias are shown in red, with yellow areas to identify clusters that also show a correlation between Negative Retrieval Success and Negative $d^{\prime}$ (inclusive masking technique thresholded at a $p<0.05$ ). Individual data points from a cluster spanning the left calcarine sulcus and lingual gyrus (circled in red in the sagittal and axial slices) are visualized in a scatter plot (bottom right). The scatted plot contains filled circles representing the $n=22$ participants included in the whole-brain analyses, but also open circles that represent the data for the seven participants excluded from the whole-brain retrieval analysis who might have noisier estimates of retrieval success activity because of a low number of misses. The red X's represent the $n=2$ participants who were not included in any of the group task or RSFC analyses but are plotted to observe how the pattern might extend if these participants were included in analysis. 
individual differences in post-encoding amygdala coupling enhancements will relate to later emotional memory biases in a valence-specific manner. To test this hypothesis, we queried the factorial model in two ways to demarcate brain regions that showed correlations between preto post-encoding increases in amygdala RSFC and emotional memory biases. We first examined the whole-brain relationship of post-encoding increases in resting state that correlated with negative memory performance $\left[\left(Z_{\text {Post }}-Z_{\text {Pre }}\right) \times\right.$ Negative $\left.d^{\prime}\right]$, but not neutral or positive memory performance [by exclusively masking out the maps of $\left(Z_{\text {Post }}-\right.$ $\left.Z_{\text {Pre }}\right) \times$ Neutral $d^{\prime}$ and $\left(Z_{\text {Post }}-Z_{\text {Pre }}\right) \times$ Positive $d^{\prime}$ each held at a reduced threshold of $p<0.05]$. Given that negative memory bias and pre- to post-encoding RSFC changes were difference measures, we further required post-encoding amygdala RSFC levels on their own to be correlated with negative memory performance (inclusively masked with the map of $Z_{\text {Post }} \times$ Negative $d^{\prime}$ ). Critically, we further report the clusters that also show a valence-specific relationship between pre- to post-encoding increases in amygdala RSFC and negative memory bias $\left[\left(Z_{\text {Post }}-Z_{\mathrm{Pre}}\right) \times\right.$ (Negative $d^{\prime}$ - Positive $d^{\prime}$ )]. That is, the greater the post-encoding increase in RSFC, the greater the difference between Negative $d^{\prime}$ and Positive $d^{\prime}$ a given participant exhibits. The reverse approach was taken to test for regions that show a relationship between post-encoding amygdala coupling increases and positive memory performance $\left[\left(Z_{\text {Post }}-Z_{\text {Pre }}\right) \times\right.$ Positive $\left.d^{\prime}\right]$ and positive memory bias $\left[\left(Z_{\text {Post }}-Z_{\mathrm{Pre}}\right) \times\right.$ Positive $d^{\prime}-$ Negative $d^{\prime}$; inclusively masked with the map of $Z_{\text {Post }} \times$ Positive $\left.d^{\prime}\right]$. We hypothesized valence-specific effects, by which parameter estimates of the slopes would not only be greater for negative compared with positive (as revealed at the whole-brain level), but also with targeted ROIs showing parameter estimates of the slope for neutral falling in the middle of negative and positive.

\section{Data reporting and visualization}

Unless otherwise specified, whole-brain group analyses were interrogated at $p<0.005$ (uncorrected). Monte Carlo simulations (https:// www2.bc.edu/sd-slotnick/scripts.htm) determined that a voxel extent of $k=40$ for memory task-fMRI analyses and $k=54$ for the resting-state fMRI analyses corrected results to $p<0.05$. As in our original study (Kark and Kensinger, 2015), all conjunction analyses to assess the replication of encoding-to-retrieval overlap were thresholded at the joint probability $p<0.005$ by setting the individual thresholds of each voxel at encoding and retrieval to $p=0.0243$ (calculated using the Fisher equation; Fisher, 1973). Because of the resolution $\left(2 \mathrm{~mm}^{3}\right)$, voxels in brainstem regions are not reported in the tables. Follow-up ROI analyses were conducted by using REX (http://web.mit.edu/swg/software.htm) to extract first-level parameter estimates for each subject for the conditionsof-interest to be entered into ANOVAs and $t$ tests and to extract the second-level parameter estimates of slopes to visualize the correlations between amygdala post-encoding RSFC by phase and memory performance by valence (see Fig. 4, bar plots). Foci conversion (Montreal Neurological Institute [MNI] and Talairach and Tournoux [TAL] coordinates) was implemented using the GingerAle (http://www. brainmap.org/ale/). Rendering of statistical maps was implemented using MRIcroGL (http://www.mccauslandcenter.sc.edu/mricrogl/home) and MRICRON (http://people.cas.sc.edu/rorden/mricron/index.html).

\section{Results}

\section{Memory performance and emotional memory bias}

Overall recognition memory performance $\left(d^{\prime}\right)$ varied across the 29 participants $(\mathrm{M}=0.74, \mathrm{SD}=0.33, \mathrm{SE}=0.06$, range: $0.17-$ $1.52)$ and was lower than in our original 20 min delay study $(\mathrm{M}=$ 1.22 , SE $=0.1$; Kark and Kensinger, 2015), which is not surprising given the longer study-test interval. As in our 2015 paper, we observed no significant group-level effects of valence on memory performance $\left(F_{(2,56)}=1.7, p=0.19\right.$, ANOVA) between negative $(\mathrm{M}=0.76, \mathrm{SD}=0.40, \mathrm{SE}=0.08)$, positive $(\mathrm{M}=0.80, \mathrm{SD}=0.44$, $\mathrm{SE}=0.08)$, and neutral stimuli $(\mathrm{M}=0.69, \mathrm{SD}=0.34, \mathrm{SE}=$ $0.06)$. Importantly, there was a range of memory bias scores (Negative $d^{\prime}-$ Positive $d^{\prime}$ ) scores across participants ( $\mathrm{SD}=0.35$, range: -0.71 to +0.71$)$; 13 participants showed a negative mem- ory bias, whereas 16 showed a positive memory bias (see full spread of negative and positive memory bias in Fig. 4, bottom, scatter plots). Individual differences in memory biases were not correlated with differences in post-scan ratings of arousal and absolute valence between the negative and positive IAPS images across the 29 participants (Arousal ${ }_{\text {neg-pos }}: r_{(27)}=0.16, p=0.41$; Absolute Valence ${ }_{\text {neg-pos }}: r_{(27)}=0.19, p=0.34$, Pearson's correlation), suggesting that they arose from memory differences and not merely differences in emotional experience.

\section{Memory task-based fMRI results}

Replication of enhanced visuosensory recapitulation for negative memories

Before examining new questions regarding individual differences in emotional memory biases, we first sought to replicate our prior findings demonstrating enhanced recapitulation in visuosensory regions for negative memories (Kark and Kensinger, 2015; Bowen and Kensinger, 2017) and confirm that enhanced grouplevel visuosensory recapitulation for negative events extends to a $24 \mathrm{~h}$ study-test delay. Indeed, the conjunction analyses revealed encoding-to-retrieval overlap for negative memories in the bilateral ventral visual stream (inferior temporal and fusiform gyri), parahippocampal cortex, parietal areas, as well as lateral and orbital portions of the prefrontal cortex (Fig. 2A, activity in red; Kark and Kensinger, 2015, their Fig. 3).

Additional control analyses show that many of these negative memory recapitulation regions, including ventral visual regions such as the inferior temporal gyri and parahippocampal cortex, remain even when the post-scan ratings of arousal were entered as a participant-specific regressor in the fixed effects models (Fig. $2 A$, activity in magenta). However, two of the ventral visual stream clusters (of the right inferior temporal gyrus clusters and of the left fusiform gyrus clusters) no longer reached significance with item-level arousal ratings in the models. In another control analysis, there were no regions that showed arousal-memory interactions across encoding and retrieval (i.e., a conjunction of positive parametric relation of arousal for Hits $>$ Misses across both phases), further suggesting the majority of these recapitulation effects were not driven by systematic differences in arousal between the valences. However, future work with tighter controls is needed to clarify valence-arousal interactions. Notwithstanding, these replication results and additional control analyses were critical for three reasons: They allowed us to (1) root the central individual differences questions of the current study in a replication of prior work (Kark and Kensinger, 2015); (2) to further demonstrate that negative valence indeed enhances recapitulation in the present paradigm with a $24 \mathrm{~h}$ study-test delay, suggesting that visuosensory enhancement remains relevant to negative memory processes long after encoding; and 3) to show that subjective arousal differences are not driving the valence differences in the distribution of the recapitulation effects.

Links between individual differences in negative memory bias and valence-specific retrieval success activity

With the recapitulation results replicated, we then moved on to ask new questions regarding links between valence-enhanced retrieval activity and emotional memory bias. Because we have consistently found group effects of visuosensory recapitulation for negative memories, we hypothesized that individual differences in negative memory bias would be associated with greater memory-related visuosensory activation at the time of retrieval. If visuosensory processes are linked with better memory for negative but not positive stimuli, those participants with greater 
success-related retrieval activity in visual processing regions will be biased toward remembering more of the negative images, compared with the positive images. To test this hypothesis, we examined the whole-brain correlations between Negative $>$ Positive Retrieval Success Activity (directional contrast) and behavioral negative memory bias (Negative $d^{\prime}-$ Positive $d^{\prime}$ ). We additionally masked this map with the relationship between Negative Retrieval Success Activity (Hits $>$ Misses) correlated with Negative $d^{\prime}$ (at $p<0.05$; Fig. $2 B$, activity in yellow), to ensure effects in the resulting clusters were not driven by an inverse correlation with positive memory. These analyses identified several visual cortex clusters including a large swath of the calcarine sulcus $\left(\mathrm{MNI}_{\mathrm{xyz}}=4,-88,-10, k=115\right)$, the left lingual gyrus $\left(\mathrm{MNI}_{\mathrm{xyz}}=-22,-82,-16, k=79\right)$, and the right occipital fusiform gyrus $\left(\mathrm{MNI}_{\mathrm{xyz}}=20,-76,-10, k=50\right)$, each with corresponding peaks that survived the inclusive masking technique $\left(\mathrm{MNI}_{\mathrm{xyz}}=-6,-86,-10, k=30 ; \mathrm{MNI}_{\mathrm{xyz}}=-20,-82\right.$, $-16, k=25 ; \mathrm{MNI}_{\mathrm{xyz}}=4,-92,-10 k=18, \mathrm{MNI}_{\mathrm{xyz}}=24,-78$, $-10, k=12)$. No other clusters outside of the visual cortex were identified by the masking procedure. The same clusters were identified when a follow-up model included an across subject covariate of post-scan rating differences of arousal between negative and positive stimuli (i.e., Arousal $_{\text {neg-pos }}$ ). There were no suprathreshold voxels for the comparison assessing a relation between positive memory bias and valence-specific retrieval activity for positive stimuli. Together, these results suggest that those participants with greater memory-related activity in visuosensory regions are also those that remember more of the negative than positive stimuli at the time of retrieval. Importantly, these effects were not driven by participants who merely thought the negative stimuli were more arousing than the positive stimuli, further suggesting a valence-specific enhancement in memory related to enhanced visuosensory memory-related activation at retrieval.

\section{Resting-state fMRI results}

Before turning to the novel aspects of the present study, we first sought to root our individual differences analyses in a replication of past work of group-level amygdala RSFC patterns (Geissmann et al., 2018). First, pre-encoding resting-state networks resembled those previously reported (Roy et al., 2009; Geissmann et al., 2018), with positive RSFC of the bilateral amygdala with large swaths of ventromedial and dorsomedial prefrontal cortex (PFC), temporal lobes, orbital and inferior PFC (Fig. 3A, red). Anti-correlations were observed in the middle frontal gyrus, parietal areas, and precuneus (Fig. $3 A$, blue). These finding suggest our group of participants show typical amygdala RSFC patterns at rest before encoding. Second, at the group-level, we found preto post-encoding increases in amygdala RSFC with regions such as the precuneus, inferior frontal gyrus, middle temporal gyrus, and insula (Table 1; Fig. 3B). We found minimal group-level preto- post-encoding increases in amygdala RSFC in visuosensory areas, with the exception of right amygdala RSFC increases with a portion of the right inferior temporal gyrus and the right temporal pole. Although these changes suggest some reconfiguration of amygdala networks detectable at the group-level following emotional picture viewing, these patterns but do not inform us about emotional memory processes specifically.

\section{Individual differences in post-encoding amygdala RSFC and} emotional memory biases

After establishing that our task effects replicate our prior work (Kark and Kensinger, 2015) and the pre-encoding amygdala

\section{A Pre-encoding (baseline) amygdala $\operatorname{RSFC}\left(Z_{\text {Pre }}\right)$}

Positive Correlations

Negative Correlations

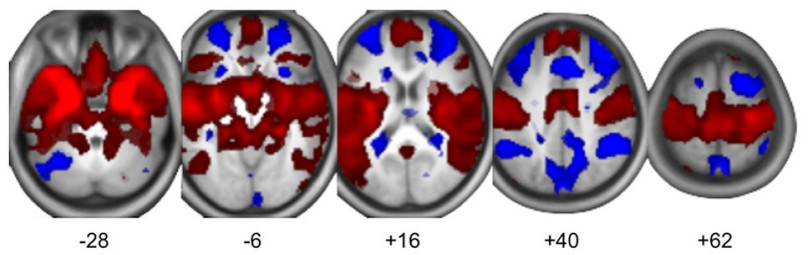

B Post-encoding $\Delta$ amygdala RSFC $\left(Z_{\text {Post }}>Z_{\text {Pre }}\right)$

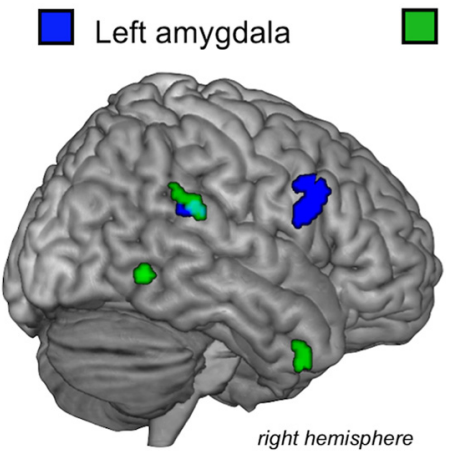

Right amygdala

Figure 3. $\quad A$, Group-average amygdala RSFC during pre-encoding (baseline) replicates a typical widespread pattern of positive correlations (red) and anti-correlations (blue). $\boldsymbol{B}$, Depicts the group-level pre- to post-encoding increases in left (blue) and right (green) amygdala RSFC. Overlap between the left and right amygdala maps in the precuneus are shown in cyan.

RSFC is consistent with previously-reported patterns (Geissmann et al., 2018), we then moved on to address the critical analyses: Examining links between individual differences in immediate post-encoding amygdala RSFC increases and long-term valence-specific emotional memory biases. We hypothesized that off-line amygdala-visuosensory and amygdala-frontal RSFC enhancements during post-encoding rest, compared with preencoding rest, would be associated with later negative and positive memory biases, respectively. Consistent with this hypothesis, negative memory performance (Negative $d^{\prime}$ ) was correlated with post-encoding enhancements of right amygdala RSFC with early visual cortex (BA17; spanning the cuneus, calcarine sulcus, occipital pole) as well as the superior, middle, and inferior occipital gyri (BA18/19; results for neutral and positive memory performance exclusively masked out; Fig. 4, activity in red; Table 2). The whole-brain analyses further demarcated two right visual regions [lingual gyrus/inferior occipital gyrus (BA18/ 19), $k=103$; inferior/middle occipital gyrus (BA19], $k=147$ )] that showed a whole-brain correlation between negative memory bias (Negative $d^{\prime}$ - Positive $d^{\prime}$ ) and post-encoding increases of right amygdala RSFC (Fig. 4, plots and activity in yellow. Table 2, see symbols to demarcate significant bias). The whole-brain RSFC effects of the left amygdala were not as wide spread as the right amygdala; however, the only cortical region to show a relation between post-encoding enhancements of left amygdala RSFC and negative memory bias was the same right lingual gyrus/ inferior occipital gyrus region $\left(\mathrm{MNI}_{\mathrm{xyz}}=+28,-88,-18, k=\right.$ 20) that showed the effect with the right amygdala.

Whereas negative memory performance and negative memory bias were associated with amygdala-visuosensory increases in RSFC, positive memory performance was associated with right amygdala-frontal post-encoding increases in RSFC, including the dorsal anterior cingulate cortex (dACC), ventrolateral PFC, infe- 
Table 1. Group pre- to post-encoding increases in amygdala RSFC

\begin{tabular}{|c|c|c|c|c|c|c|}
\hline Lobe & Region & Hemisphere & $B A$ & MNl; $x, y, z$ & $\mathrm{TAL} ; x, y, z$ & $k$ \\
\hline \multicolumn{7}{|c|}{ Right amygdala } \\
\hline \multirow[t]{4}{*}{ Frontal } & Inferior frontal gyrus & L & 47 & $-32,14,-26$ & $-30,14,-18$ & 12 \\
\hline & Precentral gyrus & $\mathrm{R}$ & 6 & $32,-8,44$ & $28,-13,43$ & 16 \\
\hline & & $\mathrm{R}$ & 6 & $30,-6,60$ & $26,-13,58$ & 13 \\
\hline & Superior frontal gyrus, supplementary motor area & $\mathrm{R}^{*}$ & 6 & $12,0,60$ & $9,-7,58$ & 42 \\
\hline \multirow[t]{4}{*}{ Parietal } & Precuneus, superior parietal lobule & $L^{*}$ & 7 & $-16,-44,54$ & $-17,-47,48$ & 104 \\
\hline & Paracentral lobule & $\mathrm{R}$ & 5 & $14,-32,52$ & $11,-36,48$ & 16 \\
\hline & Precuneus & $\mathrm{R}^{*}$ & 7 & $14,-44,54$ & $11,-48,49$ & 133 \\
\hline & Supramarginal gyrus, superior temporal gyrus & $\mathrm{R}^{*}$ & 22,40 & $56,-38,22$ & $50,-39,21$ & 68 \\
\hline \multirow[t]{2}{*}{ Temporal } & Inferior temporal gyrus & $\mathrm{R}$ & 37 & $50,-58,-8$ & $45,-55,-7$ & 37 \\
\hline & Temporal pole & $\mathrm{R}$ & 21,38 & $44,8,-42$ & $40,9,-32$ & 44 \\
\hline Other & Insula & $\mathrm{R}$ & 13 & $30,18,14$ & $27,14,19$ & 75 \\
\hline \multicolumn{7}{|l|}{ Left amygdala } \\
\hline \multirow[t]{2}{*}{ Frontal } & Inferior frontal gyrus, precentral gyrus, & $\mathrm{R}$ & 6,9 & $60,12,22$ & $54,7,26$ & 126 \\
\hline & Supplementary motor area & $\mathrm{R}^{*}$ & 6 & $10,0,56$ & $8,-7,54$ & 57 \\
\hline \multirow[t]{4}{*}{ Parietal } & Precentral gyrus & $\mathrm{L}$ & 6 & $-34,-10,36$ & $-33,-14,35$ & 16 \\
\hline & Precuneus, postcentral gyrus & $L^{*}$ & 5,7 & $-16,-44,54$ & $-17,-47,48$ & 28 \\
\hline & Precuneus, precentral and postcentral gyrus & $R^{*}$ & 7,31 & $16,-28,48$ & $13,-32,45$ & 167 \\
\hline & Supramarginal gyrus, superior temporal gyrus & $R^{*}$ & 22,40 & $46,-34,24$ & $41,-36,23$ & 166 \\
\hline Other & Parietal operculum & $\mathrm{L}$ & 13 & $-38,-30,22$ & $-37,-31,21$ & 16 \\
\hline
\end{tabular}

*Signifies overlap between the left and right amygdala maps.

\section{Post-encoding $\Delta$ in resting amygdala connectivity correlated with:}

$\square$ Negative memory performance $\square$ Positive memory performance

Negative memory bias

Positive memory bias
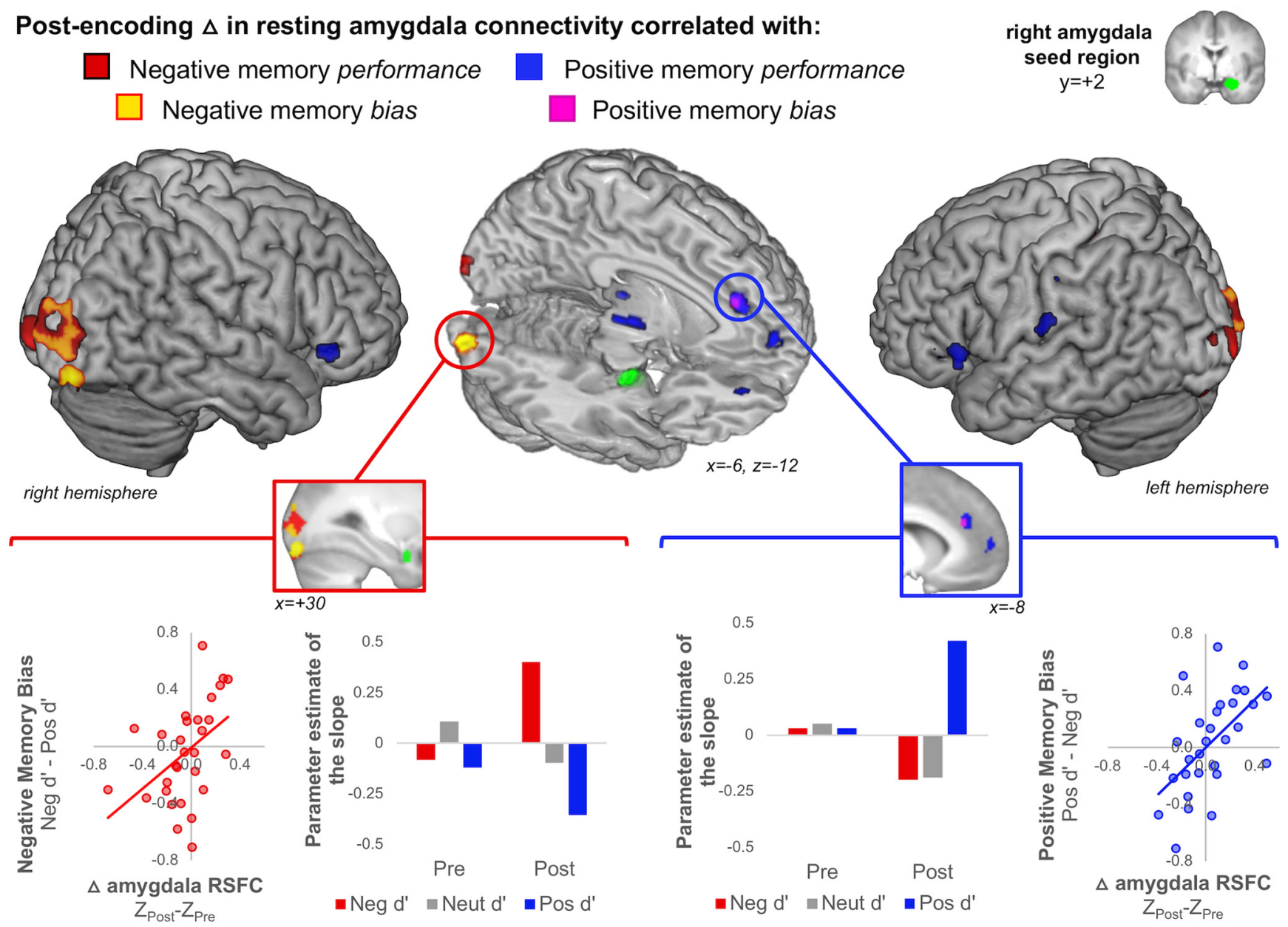

Figure 4. Post-encoding enhancement of right amygdala connectivity and valence-specific emotional memory biases. The right amygdala seed region is shown in green. Whole-brain correlations between post-encoding increases in amygdala connectivity and Negative memory performance (Negative $d^{\prime}$ ) are shown in red and Positive memory performance (Positive $d^{\prime}$ ) effects are shown in blue. Whole-brain correlations that additionally show Negative memory bias (Negative $d^{\prime}-$ Positive $d^{\prime}$ ) are shown in the inferior occipital and lingual gyri in yellow while Positive memory bias (Positive $d^{\prime}$ - Negative $d^{\prime}$ ) are shown in the dACC in violet. The bar plots display the random-effects level parameter estimates of the slope for the correlations between amygdala RSFC and memory performance by valence (negative, neutral, positive) and phase (pre-encoding, post-encoding). The scatter plots depict the relationship between the magnitude of post-encoding amygdala RSFC enhancement $\left(Z_{\text {Post }}-Z_{\text {Pre }}\right)$ and magnitude of the given emotional memory bias. 
Table 2. Across-subject correlations between post-encoding increases in right amygdala RSFC and emotional memory performance (exclusively masking out the effects for the other two valences)

\begin{tabular}{|c|c|c|c|c|c|c|}
\hline Lobe & Region & Hemisphere & $\mathrm{BA}$ & $\mathrm{MNI} ; x, y, z$ & TAL; $x, y, z$ & $k$ \\
\hline \multicolumn{7}{|c|}{ Negative memory performance } \\
\hline \multirow[t]{4}{*}{ Occipital } & Calcarine sulcus, cuneus & $\mathrm{L}$ & 17,18 & $0,-102,-6$ & $-1,-96,-10$ & 79 \\
\hline & Middle occipital gyrus & $\mathrm{L}$ & 18 & $-30,-98,8$ & $-29,-94,2$ & 11 \\
\hline & Cuneus, superior and middle occipital gyri & $R^{*}$ & $17,18,19$ & $20,-100,18$ & $17,-97,12$ & 492 \\
\hline & Lingual gyrus, inferior occipital gyrus, fusiform gyrus & $\mathrm{R}^{* a}$ & 18,19 & $28,-88,-18$ & $25,-82,-19$ & 108 \\
\hline Parietal & Postcentral gyrus & $\mathrm{L}$ & 3 & $-22,-32,52$ & $-22,-36,47$ & 60 \\
\hline Frontal & Superior frontal gyrus, supplementary motor area & $\mathrm{R}$ & 6 & $16,-4,58$ & $13,-11,56$ & 21 \\
\hline Temporal & Temporal pole & $\mathrm{R}$ & 38 & $18,8,-50$ & $16,10,-40$ & 21 \\
\hline Other & Cerebellum & $L$ & NA & $-26,-90,-26$ & $-25,-83,-27$ & 12 \\
\hline \multicolumn{7}{|c|}{ Positive memory performance } \\
\hline \multirow[t]{5}{*}{ Frontal } & Inferior frontal gyrus & $\mathrm{L}$ & 45 & $-54,22,0$ & $-51,19,5$ & 38 \\
\hline & & $\mathrm{L}$ & 46 & $-38,36,0$ & $-36,32,7$ & 11 \\
\hline & & $\mathrm{R}$ & 45 & $52,30,-4$ & $47,26,4$ & 57 \\
\hline & Superior frontal gyrus (anterior) & L & 10 & $-10,56,6$ & $-10,50,14$ & 33 \\
\hline & Orbital frontal gyrus & $\mathrm{R}$ & 11 & $10,46,-16$ & $9,42,-6$ & 13 \\
\hline \multirow[t]{5}{*}{ Other } & Cerebellum & $\mathrm{R}$ & NA & $12,-56,-38$ & $10,-51,-35$ & 34 \\
\hline & Dorsal anterior cingulate & $\mathrm{L}^{* b}$ & 32 & $-10,38,24$ & $-10,32,29$ & 104 \\
\hline & Mid-cingulate & $\mathrm{L}$ & 31 & $-2,-14,46$ & $-3,-19,44$ & 11 \\
\hline & Thalamus & $\mathrm{L}$ & NA & $-16,-14,2$ & $-16,-15,4$ & 104 \\
\hline & & $L$ & NA & $-2,-24,8$ & $-3,-25,9$ & 17 \\
\hline \multirow[t]{2}{*}{ Parietal } & Post-central gyrus & $\mathrm{L}$ & 40 & $-58,-22,20$ & $-55,-24,19$ & 63 \\
\hline & & $\mathrm{L}$ & 2 & $-46,-20,34$ & $-44,-23,32$ & 19 \\
\hline Temporal & Transverse temporal gyrus & $\mathrm{R}$ & 41 & $40,-28,4$ & $36,-28,6$ & 17 \\
\hline
\end{tabular}

Links with emotional memory biases are indicated with superscripted symbols. *Signifies whole-brain relation to emotional memory bias ( $k$ values of subclusters reported in the text).

${ }^{a}$ Left amygdala pre-to-post RSFC shows whole-brain correlation with negative memory bias $(k=20)$.

${ }^{b}$ Left amygdala pre-to-post RSFC shows whole-brain correlation with positive memory performance $(k=32)$.

rior frontal gyrus, orbital frontal cortex, and medial prefrontal cortex (Table 2). Whole-brain analyses identified voxels $(k=37)$ within the dACC as associated with positive memory bias (Table 2 , see symbol to demarcate significant bias). The same dACC region showed a whole-brain correlation between left amygdala increases in RSFC and positive memory performance $\left(\mathrm{MNI}_{\mathrm{xyz}}=\right.$ $-4,36,26, k=32$ ), but only a subthreshold relation to positive memory bias (whole-brain threshold $p=0.05, k=16$ ). Further, post-encoding RSFC increases of the left amygdala with the superior frontal gyrus showed a significant relationship to positive memory bias $\left(\mathrm{MNI}_{\mathrm{xyz}}=-22,18,38, k=16\right)$. Overall, these results suggest a similar pattern of RSFC effects of the right and left amygdala. Follow-up analyses of pre-retrieval RSFC of the right amygdala suggest no significant link between pre-retrieval amygdala-visuosensory levels of RSFC and negative memory bias $\left(r_{(26)}=-0.15, p=0.44\right.$, Pearson's correlation $)$ or amygdaladACC levels RSFC and positive memory bias $\left(r_{(26)}=0.02, p=\right.$ 0.93 , Pearson's correlation). These data suggest the principal findings are detectable shortly after encoding and there is no significant relationship between pre-retrieval configuration of the amygdala RSFC networks with these areas and valencespecific memory performance. Together, these results provide new evidence of valence-specific effects of amygdala functional connectivity enhancements with distant brain regions after encoding on subsequent behavioral memory biases.

Given prior work establishing links between memory vividness and occipital activity (Richter et al., 2016), we tested whether the present visuosensory-amygdala RSFC links with negative memory bias were also related to biases in negative memory vividness (i.e., negative memory vividness - positive memory vividness). Results returned no significant relationship between right amygdala post-encoding RSFC increases with the two visuosensory clusters that showed a relation to a greater negative memory bias (right lingual gyrus/inferior occipital gyrus: $r_{(27)}=$
$-0.07, p=0.71$; right inferior/middle occipital gyrus: ${ }_{r(27)}=$ $-0.07, p=0.73$, Pearson's correlations). Similarly, follow-up analysis returned no significant relationship between right amygdala post-encoding increases with the dACC and greater positive memory vividness, compared with negative memory vividness $\left(r_{(27)}=-0.27, p=0.16\right.$, Pearson's correlation). However, we instructed participants to make vividness ratings based not only on memory for visual details but also thoughts, feelings, or reactions to the original photo, making it impossible to draw conclusions about the content of the memoranda driving vividness for each trial. Our null results could reflect that participants use one aspect of vividness to rate negative vividness (e.g., visual details) and another aspect (e.g., thoughts or feelings) to rate positive vividness.

\section{Control analyses}

We ran several control analyses to confirm that the relationship between post-encoding increases in amygdala RSFC and negative memory biases were not driven by individual valence differences in univariate encoding levels of amygdala and visuosensory (1) activity, (2) functional connectivity, (3) "background connectivity", or (4) valence-differences in the post-scan subjective ratings of valence and arousal across participants, or (5) parallel changes in hippocampal or other subcortical connectivity with these areas. Encoding functional connectivity models were created using the gPPI toolbox (http://brainmap.wisc.edu/PPI; McLaren et al., 2012). Encoding background connectivity was calculated similar to previously reported methods (Al-Aidroos et al., 2012; Duncan et al., 2014; Murty et al., 2017) by extracting signal from the right amygdala, right lingual gyrus/inferior occipital gyrus, and dACC, from fixed-effect model residuals, which are thought to represent task and noise filtered signal. These signals were bandpass filtered $(0.01-0.08 \mathrm{~Hz})$ and pairwise correlation coefficients were $r$-to- $z$ transformed and saved as the metric of background connectivity 


\section{A Exploratory mediation analysis}

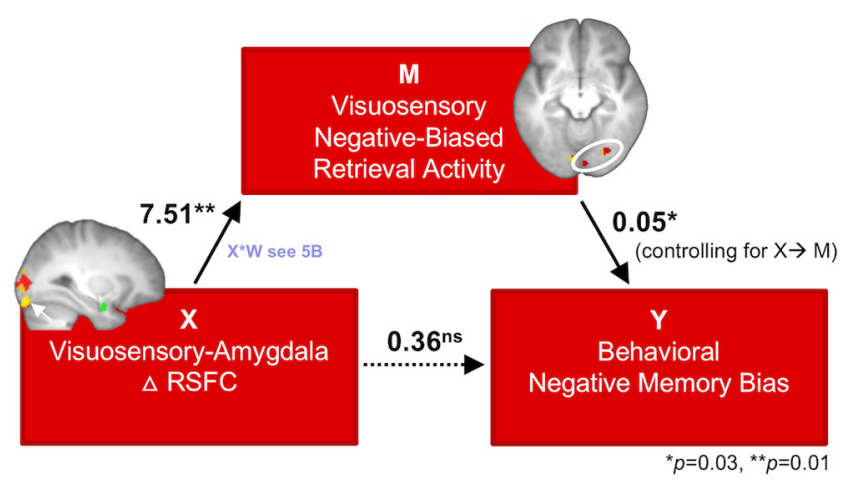

Total effect, $b=0.73, \mathrm{SE}=0.28, p=0.02,95 \% \mathrm{Cls}=[0.15,1.32]$

Direct effect, $b=0.38, \mathrm{SE}=0.30, p=0.22,95 \% \mathrm{Cls}=[-0.24,0.99]$

Indirect effect, $\mathrm{b}=0.36, \mathrm{SE}_{\mathrm{boot}}=0.20,95 \% \mathrm{Cls}_{\text {boot }}=[0.08,0.87]$

\section{B Modulatory effect of Visuosensory-Hippocampal $\triangle$ RSFC}

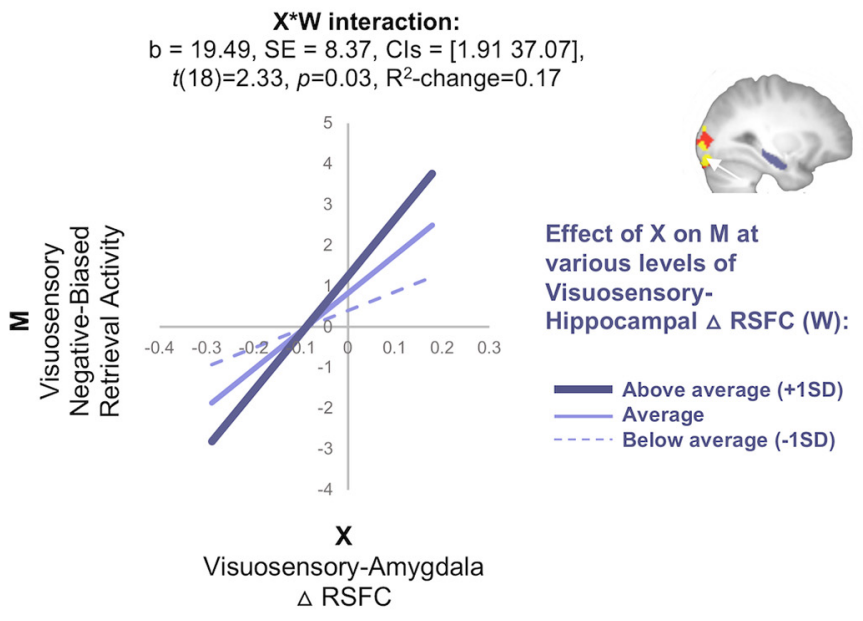

Figure 5. A, Exploratory Mediation analysis. Visuosensory Negative-Biased Retrieval Activity in right occipital cortex (average of two right hemisphere visuosensory regions shown in the axial slice, top) completely mediated the relationship between pre- to post-encoding increases in Visuosensory-Amygdala RSFC and Behavioral Negative Memory Bias, which suggests post-encoding amygdala-visuosensory RSFC enhancements set up the brain for a negative-biased retrieval mode visuosensory regions at the time of retrieval. The path values represent the unstandardized regression coefficients. Significance of the indirect effect was determined by the bootstrapped $95 \% \mathrm{Cl}(10,000$ samples). $\boldsymbol{B}$, Additional first-stage moderated mediation analysis. Follow-up analysis of pre- to post-encoding changes of the right hippocampus and the right visuosensory region (shown in sagittal slice) revealed a significant moderated mediation, whereby the effect of post-encoding amygdala-visuosensory RSFC on Visuosensory Negative-Biased Retrieval Activity depended on the level of pre- to post-encoding changes of visuosensory-hippocampal RSFC. The significant $X \times W$ interaction is plotted at various levels of visuosensory-hippocampal changes in RSFC. ${ }^{*} p=0.03,{ }^{* *} p=0.01$.

for each participant. These control metrics of interest were entered as covariates in four separate follow-up random-effects models to confirm that the whole-brain links between individual differences in pre- to post-encoding increases in right amygdala RSFC and emotional memory biases remained suprathreshold when controlling for these possible across-subject explanations of the effects. The principal valence-specific links of right amygdala RSFC links with negative and positive memory biases remained significant in the inferior occipital gyrus and dACC, respectively, when these additional random-effects factorial models controlled for (1) encoding activity differences between negative and positive hits and (2) functional connectivity differences in the right amygdala, right lingual gyrus/inferior occipital gyrus, and dACC between negative and positive hits, as well as (3) encoding background connectivity differences of the RAMY with these target regions, and (4) valence differences in post-scan ratings of arousal and absolute valence (i.e., Arousal neg-pos $_{\text {- }}$ ). RSFC analysis of other subcortical seed regions (i.e., hippocampus and putamen) with the principal visuosensory clusters associated with negative memory biases returned no significant link with negative memory bias (all $r$ values $<0.3$, $p$ values $>0.1$, Pearson's correlations). A similar pattern was observed for positive memory bias and post-encoding RSFC increases with the dACC cluster. These data suggest the principal negative memory bias effects might be specific to the amygdala and were not driven by hippocampal (but see Exploratory mediation analysis) or putamen influences of increased post-encoding RSFC with these cortical targets.

Follow-up analysis of post-encoding resting-state fMRI scans available from the original 20 min study-test delay study (Kark and Kensinger, 2015) using a separate set of participants shows no significant correlation between post-encoding right amygdalavisuosensory RSFC and negative memory bias $\left(r_{(20)}=-0.24\right.$, $p=0.28$, Pearson's correlation) or right amygdala-dACC RSFC and positive memory bias $\left(r_{(20)}=-0.1, p=0.67\right.$, Pearson's correlation). These data suggest that a delay $>20$ min might be required to observe a relation between post-encoding amygdala
RSFC increase with these ROIs and long-term memory. However, future work is needed to directly confirm the need for an extended delay, because these RSFC scans were collected on a different scanner and used different acquisition parameters (47 slices, TR $=3000 \mathrm{~ms}, 100$ images, $3 \mathrm{~mm}^{3}$ voxels) and preencoding scans were not collected. Together, these follow-up analyses demonstrate that the principal findings are not driven by differences during encoding or by subjective reactivity differences to the images as valence categories, and likely reflect enhanced amygdala engagement following encoding that is relevant to later behavior in a valence-specific fashion.

\section{Exploratory mediation analysis}

We have presented evidence that negative memory biases are associated with visuosensory engagement both online during retrieval and off-line during post-encoding rest periods: Specifically, we have demonstrated that negative memory bias is related to both (1) greater retrieval success activation in visuosensory areas for negative compared with positive stimuli, as well as (2) greater enhancements of visuosensory-amygdala connectivity during post-encoding off-line rest periods. Although these visuosensory areas that showed a significant relation with negative memory bias were not directly spatially overlapping, possibly because of differences of engagement while at rest compared with during task or after a period of consolidation, we use these metrics to capture individuals who show a visuosensory tendency in the brain with regard to negative memory bias. With these across-subjects metrics, we conducted an exploratory, post hoc mediation analysis to test whether post-encoding amygdalavisuosensory RSFC increases (Fig. 4) and visuosensory negativebiased retrieval activity (Fig. $2 B$ ) independently influence behavioral negative memory bias (unmediated) or whether postencoding right amygdala RSFC increases set-up visuosensory brain areas for a negative memory bias mode at retrieval (Fig. 5, Model 1). That is, do changes in amygdala-visual RSFC immediately following encoding (independent $X$ variable) influence neg- 
ative memory bias (dependent $Y$ variable) via biases in visual retrieval activity (mediator $Z$ variable)?

\section{Mediation methods}

For the Visuosensory-Amygdala $\triangle$ RSFC variable $(X)$, we chose the magnitude of right amygdala post-encoding change with the right lingual gyrus/inferior occipital gyrus region $\left(\mathrm{MNI}_{\mathrm{xyz}}=28\right.$, -88 , -16; Figs. 4, pop-out plot, yellow, 5, sagittal slice). We chose this particular area because it (1) fell nearer to the right hemisphere clusters that showed visuosensory negative-biased retrieval activity (Fig. $2 B$ ), and (2) because it fell nearer to the terminal area of the inferior longitudinal fasciculus (Catani et al., 2003), which structurally connects the amygdala and occipital cortex. For the Visuosensory Negative-Biased Retrieval Activity variable $(M)$, we averaged the parameter estimates (Negative Retrieval Success $>$ Positive Retrieval Success directional contrasts) from the right hemisphere clusters that showed a correlation with behavioral negative memory bias $\left(\mathrm{MNI}_{\mathrm{xyz}}=24,-78,-10\right.$ and $\mathrm{MNI}_{\mathrm{xyz}}=4,-92,-10$; Fig. 5 , circled on the axial slice), because amygdala effects tend to be strongest ipsilaterally (Amaral et al., 2003; Kilpatrick and Cahill, 2003; Vuilleumier et al., 2004).

We then tested the hypothesized mediation model (Fig. 5A, Model 1), with the metric of Behavioral Negative Memory Bias (Negative $d^{\prime}-$ Positive $d^{\prime}$ ) as the dependent variable $(Y)$. In Model 1, post-encoding amygdala-visuosensory RSFC increases ( $X$, Visuosensory-Amygdala $\triangle$ RSFC) predict Behavioral Negative Memory Bias via Visuosensory Negative-Biased Retrieval Activity $(M)$. In Model 2, Visuosensory NegativeBiased Retrieval Activity predicts Behavioral Negative Memory Bias via Visuosensory-Amygdala $\triangle$ RSFC on the prior day.

We used regression analysis and a bootstrapping estimation method to determine significance of the mediation model using PROCESS (http://www.processmacro.org/index.html; Hayes, 2018), regression path analyses modeling tool we implemented in IBM SPSS Statistics 24. Mediation is determined significant if the confidence interval does not contain 0 . Unstandardized regression coefficients ( $b$ values) and bootstrapped $95 \%$ confidence intervals (10,000 iterations) were used to determine significance and standardized coefficients $(\beta)$ are reported for comparisons across studies.

\section{Mediation results}

In Model 1 (Fig. 5A), Visuosensory Amygdala Change in RSFC $(X)$ was a significant predictor of Visuosensory Negative-Biased Retrieval Success Activity $[b=7.51, \mathrm{SE}=2.79, p=0.01, \mathrm{CI}=$ $(1.69,13.34), \beta=0.52]$ and explained $27 \%$ of the variability of retrieval activity $\left(R^{2}=0.27\right)$. Visuosensory Amygdala Change in RSFC no longer predicted negative memory bias $[b=0.38, \mathrm{SE}=$ $0.30, p=0.22, \mathrm{CI}=(-0.24,0.99), \beta=0.26]$ when Visuosensory Negative-Biased Retrieval Success bias was added to the model as a mediator $[b=0.05, \mathrm{SE}=0.02, p=0.03, \mathrm{CI}=(0.01,0.09), \beta=$ 0.48 ], suggesting a complete mediation. The indirect effect was found to be significant using a bootstrap estimation approach with 10,000 samples $\left[\mathrm{b}=0.36, \mathrm{SE}_{\mathrm{boot}}=0.20, \mathrm{CI}_{\mathrm{boot}}=(0.08\right.$, $0.87), \beta=0.25]$. That is, a $1 \mathrm{SD}$ difference in post-encoding right amygdala RSFC was associated with 0.1 greater difference in negative memory bias (Negative $d^{\prime}$ - Positive $d^{\prime}$ ), as mediated by visuosensory retrieval success activity. Approximately $42 \%$ of the variance in behavioral negative memory bias was accounted for by these two predictors $\left(R^{2}=0.42\right)$. The indirect effects were also significant and indicated a full mediation when additional models were run with the two retrieval clusters averaged to create the $M$ variable were run separately [when $M$ values were extracted from $\mathrm{MNI}_{\mathrm{xyz}}=4,-92,-10$ : $b=0.35, \mathrm{SE}_{\text {boot }}=0.20,95 \% \mathrm{CI}_{\mathrm{boot}}=(0.07,0.88), \beta=0.24$; $\mathrm{MNI}_{\mathrm{xyz}}=24,-78,-10: b=0.29, \mathrm{SE}_{\mathrm{boot}}=0.17,95 \% \mathrm{CI}_{\mathrm{boot}}=$ $(0.04,0.71), \beta=0.20]$, suggesting that neither cluster drove the indirect effect when collapsed across those two clusters. A full mediation suggests post-encoding enhancements of Visuosensory-Amygdala $\triangle$ RSFC predict later Visuosensory Negative-Biased Retrieval Activity, which in turn predicts the magnitude of the Behavioral Negative Memory Bias. In other words, post-encoding processes set up the brain for a negativebiased retrieval mode, which drives the gap between remembering more of the bad than the good.

Although Model 1 makes the most sense both theoretically and chronologically (i.e., post-encoding processes precede retrieval activity), we also evaluated the possibility that the association between retrieval activity bias and negative memory bias could be mediated by a prior history of post-encoding increases in amygdala RSFC, and not that amygdala RSFC increases cause retrieval activity biases. Using the same approach but with Visuosensory Negative-Biased Retrieval Activity as the independent variable $(X)$ and Visuosensory-Amygdala $\triangle$ RSFC as the mediator $(M)$, the indirect effect in Model 2 was not found to be significant $\left[b=0.01, \mathrm{SE}_{\text {boot }}=0.01, \mathrm{CI}_{\text {boot }}=(-0.01,0.04), \beta=0.13\right]$.

\section{Additional first-stage moderated mediation model}

We further explored the possibility of hippocampal contributions to these links. Although post-encoding increases in right hippocampal RSFC (Visuosensory-Hippocampal $\triangle$ RSFC) with the right lingual gyrus/inferior occipital gyrus region were not statistically significant $\left(r_{(27)}=0.28, p=0.14\right.$, Pearson's correlation), post-encoding increases of Visuosensory-Hippocampal $\triangle$ RSFC moderated the relationship between VisuosensoryAmygdala $\triangle$ RSFC and Visuosensory Negative-Biased Retrieval Activity (Fig. 5B, plot of the interaction). A moderated mediation model further revealed that the indirect effect of $X$ on $Y$ through $M$ shown in Figure $5 A$ was only significant at average or greater-than-average levels of VisuosensoryHippocampal $\triangle \mathrm{RSFC}\left[\mathrm{W}_{\text {average-1SD }}: b=0.22, \mathrm{SE}_{\mathrm{boot}}=0.22\right.$, $\mathrm{CI}_{\text {boot }}=(-0.25,0.64) ; \mathrm{W}_{\text {average }}: b=0.44, \mathrm{SE}_{\text {boot }}=0.26$, $\mathrm{CI}_{\text {boot }}=(0.02,1.05) ; \mathrm{W}_{\text {average }+1 \mathrm{SD}}: b=0.67, \mathrm{SE}_{\text {boot }}=$ $\left.0.43, \mathrm{CI}_{\text {boot }}=(0.10,1.68)\right]$. Although these findings require further examination with a larger sample size, these results provide preliminary evidence that greater visuosensoryhippocampal increases in post-encoding RSFC augment the indirect effect that visuosensory-amygdala RSFC exerts on negative memory biases.

\section{Discussion}

The present study is the first to demonstrate not only valencespecific emotional memory retrieval patterns but also valencespecific links between post-encoding increases in amygdala-cortical RSFC and long-term episodic emotional memory biases. These results emphasize that negative memories differ from positive not only in the way the content is initially encoded but also in how that content is consolidated over time and brought to mind at retrieval. Primarily, we demonstrate that behavioral negative memory bias was specifically associated with off-line postencoding RSFC increases of the amygdala with occipital areas, and that activation of similar visual regions at the time of retrieval was also linked to negative memory bias.

These findings are consistent with a growing body of work demonstrating that immediate post-learning functional connectivity of the MTL can have long-term consequences on subsequent memory 
(Tambini et al., 2010, 2017; Staresina et al., 2013; Tambini and Davachi, 2013), including fear memory (de Voogd et al., 2016; Hermans et al., 2017). Here we show that some of these effects are dissociable along the dimension of emotional valence. These results may provide new avenues for understanding or remediating negative memory biases, by revealing that negative memory biases can be linked to the way that sensory processes are integrated into amygdala-centered emotional memory networks.

\section{Post-encoding amygdala-cortical RSFC predicts emotional memory}

The key finding was a valence-based dissociation in the link between post-encoding amygdala RSFC increases and long-term emotional memory biases: greater amygdala engagement with occipital and frontal areas immediately following encoding was differentially associated with greater negative and positive memory biases, respectively. Inferior occipital and medial frontal areas show dense reciprocal connections with the amygdala along the inferior longitudinal fasciculus and uncinate fasciculus, respectively (Catani et al., 2003; Ghashghaei et al., 2007), making these off-line functional changes in connectivity anatomically plausible. While we can only speculate why the individual differences in negative memory biases play out in more posterior visual processing regions, one possibility is that participants with a stronger negative memory bias are bringing to mind more fine-grained visual features of the negative stimuli to support memory. Similar areas of the inferior middle occipital gyrus have been associated with sensitivity to spatial frequency information (Rotshtein et al., 2007) and retrieval of color (Ueno et al., 2007), raising the possibility that those participants with greater posterior visuosensory engagement bring to mind these visual features. In contrast, group-level recapitulation effects in relatively more anterior visual regions might reflect reactivation of higher-order visual representations (Wheeler and Buckner, 2003).

Positive memory performance was associated with postencoding amygdala RSFC increases with frontal regions, with a specific positive memory bias effect associated with amygdaladACC RSFC. These findings are broadly consistent with prior word (Mickley Steinmetz et al., 2010) but extend amygdalafrontal influences on memory into post-encoding periods. Perhaps amygdala enhancement of frontal areas involved in gist- or heuristic-based memory processing associated with positive stimuli (Kensinger and Schacter, 2008) and the experience of positive emotions (Ashby et al., 1999; Fredrickson and Branigan, 2005) tips the scale toward overall better recognition memory of positive stimuli.

\section{Amygdala-visuosensory RSFC may influence negative memory bias via retrieval activity}

The exploratory mediation analysis suggests those participants with greater post-encoding amygdala-visuosensory RSFC have greater memory-related visuosensory activity for negative stimuli compared with positive stimuli during retrieval, resulting in a more pronounced negative memory bias. Although these visuosensory areas were not directly overlapping, individuals with a tendency toward visuosensory engagement may exhibit a greater negative memory bias. The change over time in the exact visuosensory areas could plausibly be consistent with systems consolidation, such that initial changes in one set of regions may trigger changes over time in distal regions. The exploratory moderated mediation result was broadly consistent with a systems consolidation view as well, suggesting a role for the hippocampus in moderating the indirect effects of amygdala-visuosensory RSFC on negative memory bias through retrieval activity. Although exploratory, these results may help to guide further research that can settle recent debates about the role of amygdala-hippocampal interactions in emotional memory (Yonelinas and Ritchey, 2015; Inman et al., 2018).

\section{Modulatory role of the amygdala}

Our study provides new evidence in humans that in the minutes following an emotional experience, long-term behavioral emotional memory outcomes are influenced by increases in postencoding amygdala coupling with neocortical regions, and the regions of interaction determine the dominant valence to-be remembered later. The present findings are broadly consistent with the modulation hypothesis of amygdala function and are also aligned with Müller and Pilzecker's (1900) perseveration-consolidation hypothesis, which proposed that neural activity can continue for hours after initial learning, suggesting a role of the amygdala after encoding (McGaugh, 2005). Pelletier et al. (2005) suggest "that the memory-modulating role of the basolateral amygdala would not depend on the specific activation of particular groups of basolateral amygdala neurons, but on the activity patterns taking place in basolateral amygdala projection sites when the emotional arousal occurred." Yet the present results also provide intriguing evidence that, although emotional arousal undoubtedly enhances amygdala activation and engagement with distant brain regions, the target sites of those interactions may depend on valence (Tye, 2018).

\section{Limitations and future work}

There are a few limitations and important next steps in this research. Although these data are consistent with memory consolidation theories and could reflect early consolidation processes, future work is needed to confirm that RSFC changes reflect offline memory consolidation processes. Future work is needed to formally test in a within-subjects design if the link between postencoding amygdala RSFC and valence-specific memory biases require a long period of consolidation to be observed. Although RSFC studies provide an important window into memory consolidation, it is challenging to separate off-line consolidation effects from those elicited by participants' thoughts or rehearsals following encoding. For instance, it is possible that the amygdala-frontal RSFC connectivity increase corresponds with positive memory biases not because of changes to intrinsic network connectivity but because those participants who use an active emotion regulation strategy post-encoding end up remembering more of the good than the bad. Future work is needed to adjudicate between consolidation and other rehearsal or regulatory accounts of these data. Notwithstanding, the current results provide the first evidence that amygdalavisuosensory coupling following an event predicts negative memory bias and further highlights the need for valence-based accounts of emotional memory.

More generally, it will be important for future research to examine whether the pattern of results revealed here requires a longer delay. If these results reflect systems consolidation, then the link between emotional memory bias and post-encoding amygdala-neocortical interactions might depend on a lengthy study-test delay. In the fear conditioning literature, it is broadly accepted that long-term systems consolidation memory is assessed over days and weeks rather than minutes or hours (Nader, 2003; Dudai et al., 2015), yet shorter delays are common when assessing episodic emotional memory. Future episodic emotional memory work could consider that same-day testing might not be ideal for examining how "long-term" emotional episodic memory effects are instantiated in neocortical areas. 
Another direction for future research will be to clarify which aspects of memory are enhanced via these interactions with the amygdala. Recent work has emphasized differences in the effects of negative valence on subjective memory vividness, the precision of visual feature encoding, and the precision of visual retrieval (Cooper et al., 2019). Future work can test whether postencoding MTL-visuosensory interactions bear influence not only on memory discrimination, as revealed here, but also on memory measures such as visual specificity (Kensinger and Schacter, 2007; Leal et al., 2014), continuous color (Richter et al., 2016), or salience judgements (Cooper et al., 2019), that might underlie differences between negative and positive memories.

Although the present work lays a preliminary foundation to explain variability in emotional memory biases, none of the participants in the present study reported a history of affective disorders, so it will be important to decipher whether these basic valence-specific memory mechanisms map onto the exaggerated negative memory biases observed in psychopathology (Haas and Canli, 2008) or to the positive memory biases in aging that rely more heavily on prefrontal and cingulate engagement (Kensinger and Schacter, 2008), each of which can be maintained over many months.

\section{Conclusions}

The current study is the first to demonstrate that post-encoding amygdala RSFC patterns are linked with behavioral measures of valence-specific emotional memory biases. The dominant valence remembered by an individual depends on the regions showing the strongest RSFC with the amygdala post-encoding, with posterior visuosensory and frontal connectivity differentially supporting negative and positive memory biases, respectively. We circumvented and controlled for stimulus-bound differences, reducing the likelihood that these valence effects would be driven by low-level visual differences or greater subjective feelings of arousal for negative images. These findings suggest that valence-specific effects occur outside the context of encoding or retrieval tasks during off-line periods following encoding; possibly contributing to early consolidation processes. These data support a new valence-based account of emotional memory enhancement (Bowen et al., 2018) and provide evidence for valence-specific differences in amygdala connectivity that give rise to remembering more of the bad than the good.

\section{References}

Al-Aidroos N, Said CP, Turk-Browne NB (2012) Top-down attention switches coupling between low-level and high-level areas of human visual cortex. Proc Natl Acad Sci U S A 109:14675-14680.

Amaral DG, Behniea H, Kelly JL (2003) Topographic organization of projections from the amygdala to the visual cortex in the macaque monkey. Neuroscience 118:1099-1120.

Ashby FG, Isen AM, Turken AU (1999) A neuropsychological theory of positive affect and its influence on cognition. Psychol Rev 106:529-550.

Barth M, Breuer F, Koopmans PJ, Norris DG, Poser BA (2016) Simultaneous multislice (SMS) imaging techniques. Magn Reson Med 75:63-81.

Bowen HJ, Kensinger EA (2017a) Recapitulation of emotional source context during memory retrieval. Cortex 91:142-156.

Bowen HJ, Kensinger EA (2017b) Memory-related functional connectivity in visual processing regions varies by prior emotional context. Neuroreport 28:808-813.

Bowen HJ, Kark SM, Kensinger EA (2018) NEVER forget: negative emotional valence enhances recapitulation. Psychon Bull Rev 25:870-891.

Cahill L, McGaugh JL (1998) Mechanisms of emotional arousal and lasting declarative memory. Trends Neurosci 21:294-299.

Catani M, Jones DK, Donato R, Ffytche DH (2003) Occipito-temporal connections in the human brain. Brain 126:2093-2107.

Cooper RA, Kensinger EA, Ritchey M (2019) Memories fade: the relation- ship between memory vividness and remembered visual salience. Psychol Sci, in press.

de Voogd LD, Fernández G, Hermans EJ (2016) Awake reactivation of emotional memory traces through hippocampal-neocortical interactions. Neuroimage 134:563-572.

Dringenberg HC, Kuo MC, Tomaszek S (2004) Stabilization of thalamocortical long-term potentiation by the amygdala: cholinergic and transcription-dependent mechanisms. Eur J Neurosci 20:557-565.

Dudai Y, Karni A, Born J (2015) The consolidation and transformation of memory. Neuron 88:20-32.

Duncan K, Tompary A, Davachi L (2014) Associative encoding and retrieval are predicted by functional connectivity in distinct hippocampal area CA1 pathways. J Neurosci 34:11188-11198.

Feinberg DA, Moeller S, Smith SM, Auerbach E, Ramanna S, Gunther M, Glasser MF, Miller KL, Ugurbil K, Yacoub E (2010) Multiplexed echo planar imaging for sub-second whole brain FMRI and fast diffusion imaging. PloS One 5:e15710.

Fisher R (1973) Statistical methods for research workers, Ed 14. New York: Hafner.

Fox MD, Snyder AZ, Vincent JL, Corbetta M, Van Essen DC, Raichle ME (2005) The human brain is intrinsically organized into dynamic, anticorrelated functional networks. Proc Natl Acad Sci U S A 102:9673-9678.

Fox MD, Snyder AZ, Zacks JM, Raichle ME (2006) Coherent spontaneous activity accounts for trial-to-trial variability in human evoked brain responses. Nat Neurosci 9:23-25.

Fredrickson BL, Branigan C (2005) Positive emotions broaden the scope of attention and thought-action repertoires. Cogn Emot 19:313-332.

Geissmann L, Gschwind L, Schicktanz N, Deuring G, Rosburg T, Schwegler K, Gerhards C, Milnik A, Pflueger MO, Mager R, de Quervain DJF, Coynel D (2018) Resting-state functional connectivity remains unaffected by preceding exposure to aversive visual stimuli. Neuroimage 167:354-365.

Gerritsen L, Rijpkema M, van Oostrom I, Buitelaar J, Franke B, Fernández G, Tendolkar I (2012) Amygdala to hippocampal volume ratio is associated with negative memory bias in healthy subjects. Psychol Med 42:335343.

Ghashghaei HT, Hilgetag CC, Barbas H (2007) Sequence of information processing for emotions based on the anatomic dialogue between prefrontal cortex and amygdala. Neuroimage 34:905-923.

Haas BW, Canli T (2008) Emotional memory function, personality structure and psychopathology: a neural system approach to the identification of vulnerability markers. Brain Res Rev 58:71-84.

Hamann SB, Ely TD, Grafton ST, Kilts CD (1999) Amygdala activity related to enhanced memory for pleasant and aversive stimuli. Nat Neurosci 2:289-293

Hammers A, Allom R, Koepp MJ, Free SS, Myers R, Lemieux L, Mitchell TN, Brooks DJ, Duncan JS (2003) Three-dimensional maximum probability atlas of the human brain, with particular reference to the temporal lobe. Hum Brain Mapp 19:224-247.

Hayes AF (2018) Introduction to mediation, moderation, and conditional process analysis, Ed 2. New York: Guilford.

Hermans EJ, Battaglia FP, Atsak P, de Voogd LD, Fernández G, Roozendaal B (2014) How the amygdala affects emotional memory by altering brain network properties. Neurobiol Learn Mem 112:2-16.

Hermans EJ, Kanen JW, Tambini A, Fernández G, Davachi L, Phelps EA (2017) Persistence of amygdala-hippocampal connectivity and multi-voxel correlation structures during awake rest after fear learning predicts long-term expression of fear. Cereb Cortex 27:3028-3041.

Inman CS, Manns JR, Bijanki KR, Bass DI, Hamann S, Drane DL, Fasano RE, Kovach CK, Gross RE, Willie JT (2018) Direct electrical stimulation of the amygdala enhances declarative memory in humans. Proc Natl Acad Sci U S A 115:98-103.

Itti L, Koch C (2001) Computational modelling of visual attention. Nat Rev Neurosci 2:194-203

Kark SM, Kensinger EA (2015) Effect of emotional valence on retrievalrelated recapitulation of encoding activity in the ventral visual stream. Neuropsychologia 78:221-230.

Kensinger EA, Schacter DL (2007) Remembering the specific visual details of presented objects: neuroimaging evidence for effects of emotion. Neuropsychologia 45:2951-2962.

Kensinger EA, Schacter DL (2008) Neural processes supporting young and older adults' emotional memories. J Cogn Neurosci 20:1161-1173.

Kilpatrick L, Cahill L (2003) Amygdala modulation of parahippocampal 
and frontal regions during emotionally influenced memory storage. Neuroimage 20:2091-2099.

Lang PJ, Bradley MM, Cuthbert BN (2008) International affective picture system (IAPS): affective ratings of pictures and instruction manual. In: Technical report A-6. Gainesville, FL: University of Florida.

Leal SL, Tighe SK, Jones CK, Yassa MA (2014) Pattern separation of emotional information in hippocampal dentate and CA3. Hippocampus 24:1146-1155.

McGaugh JL (2000) Memory: a century of consolidation. Science 287: $248-251$.

McGaugh JL (2005) Emotional arousal and enhanced amygdala activity: new evidence for the old perseveration-consolidation hypothesis. Learn Mem 12:77-79.

McLaren DG, Ries ML, Xu G, Johnson SC (2012) A generalized form of contextdependent psychophysiological interactions (gPPI): a comparison to standard approaches. Neuroimage 61:1277-1286.

Mickley Steinmetz KR, Addis DR, Kensinger EA (2010) The effect of arousal on the emotional memory network depends on valence. Neuroimage 53:318-324.

Moeller S, Yacoub E, Olman CA, Auerbach E, Strupp J, Harel N, Uğurbil K (2010) Multiband multislice GE-EPI at 7 tesla, with 16-fold acceleration using partial parallel imaging with application to high spatial and temporal whole-brain fMRI. Magn Reson Med 63:1144-1153.

Müller GE, Pilzecker A (1900) Experimentalle beitrage zur lehre vom gedac̈htnis. Zeitschrift fuer Psychologie 1:1-288.

Murty VP, Ritchey M, Adcock RA, LaBar KS (2011) Reprint of: fMRI studies of successful emotional memory encoding: a quantitative meta-analysis. Neuropsychologia 49:695-705.

Murty VP, Tompary A, Adcock RA, Davachi L (2017) Selectivity in postencoding connectivity with high-level visual cortex is associated with reward-motivated memory. J Neurosci 37:537-545.

Nader K (2003) Memory traces unbound. Trends Neurosci 26:65-72.

Paller KA, Wagner AD (2002) Observing the transformation of experience into memory. Trends Cogn Sci 6:93-102.

Pelletier JG, Likhtik E, Filali M, Paré D (2005) Lasting increases in basolateral amygdala activity after emotional arousal: implications for facilitated consolidation of emotional memories. Learn Mem 12:96-102.

Power JD, Barnes KA, Snyder AZ, Schlaggar BL, Petersen SE (2012) Spurious but systematic correlations in functional connectivity MRI networks arise from subject motion. Neuroimage 59:2142-2154.

Richter FR, Cooper RA, Bays PM, Simons JS (2016) Distinct neural mechanisms underlie the success, precision, and vividness of episodic memory. eLife 5:e18260.

Rotshtein P, Vuilleumier P, Winston J, Driver J, Dolan R (2007) Distinct and convergent visual processing of high and low spatial frequency information in faces. Cereb Cortex 17:2713-2724.

Roy AK, Shehzad Z, Margulies DS, Kelly AM, Uddin LQ, Gotimer K, Biswal BB, Castellanos FX, Milham MP (2009) Functional connectivity of the human amygdala using resting state fMRI. Neuroimage 45:614-626.

Silverstein DN, Ingvar M (2015) A multi-pathway hypothesis for human visual fear signaling. Front Syst Neurosci 9:101.

Staresina BP, Alink A, Kriegeskorte N, Henson RN (2013) Awake reactivation predicts memory in humans. Proc Natl Acad Sci US A 110: 21159-21164.

Tambini A, Davachi L (2013) Persistence of hippocampal multivoxel patterns into postencoding rest is related to memory. Proc Natl Acad Sci U S A 110:19591-19596.

Tambini A, Ketz N, Davachi L (2010) Enhanced brain correlations during rest are related to memory for recent experiences. Neuron 65:280-290.

Tambini A, Rimmele U, Phelps EA, Davachi L (2017) Emotional brain states carry over and enhance future memory formation. Nat Neurosci 20:271-278

Tye KM (2018) Neural circuit motifs in valence processing. Neuron 100:436-452.

Ueno A, Abe N, Suzuki M, Hirayama K, Mori E, Tashiro M, Itoh M, Fujii T (2007) Reactivation of medial temporal lobe and occipital lobe during the retrieval of color information: a positron emission tomography study. Neuroimage 34:1292-1298.

Vuilleumier P, Richardson MP, Armony JL, Driver J, Dolan RJ (2004) Distant influences of amygdala lesion on visual cortical activation during emotional face processing. Nat Neurosci 7:1271-1278.

Wagner AD, Koutstaal W, Schacter DL (1999) When encoding yields remembering: insights from event-related neuroimaging. Philos Trans R Soc Lond B Biol Sci 354:1307-1324.

Waheed SH, Mirbagheri S, Agarwal S, Kamali A, Yahyavi-Firouz-Abadi N, Chaudhry A, DiGianvittorio M, Gujar SK, Pillai JJ, Sair HI (2016) Reporting of resting-state functional magnetic resonance imaging preprocessing methodologies. Brain Connect 6:663-668.

Wheeler ME, Buckner RL (2003) Functional dissociation among components of remembering: control, perceived oldness, and content. J Neurosci 23:3869-3880.

Whitfield-Gabrieli S, Nieto-Castanon A (2012) Conn: a functional connectivity toolbox for correlated and anticorrelated brain networks. Brain Connect 2:125-141.

Xu J, Moeller S, Auerbach EJ, Strupp J, Smith SM, Feinberg DA, Yacoub E, Uğurbil K (2013) Evaluation of slice accelerations using multiband echo planar imaging at 3 T. Neuroimage 83:991-1001.

Yonelinas AP, Ritchey M (2015) The slow forgetting of emotional episodic memories: an emotional binding account. Trends Cogn Sci 19:259-267. 